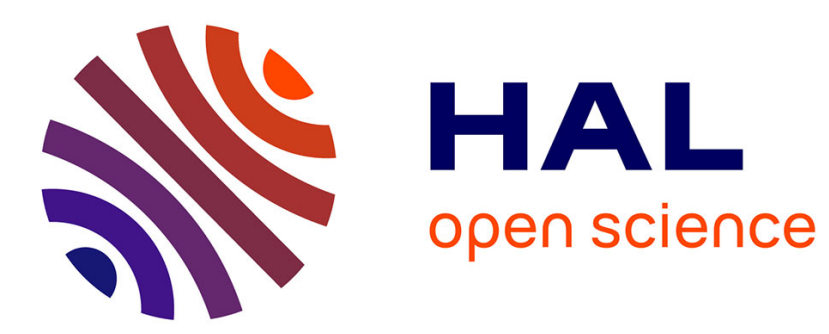

\title{
Physics with REX-ISOLDE: from experiment to facility
}

\author{
P van Duppen, K Riisager
}

\section{To cite this version:}

$\mathrm{P}$ van Duppen, K Riisager. Physics with REX-ISOLDE: from experiment to facility. Journal of Physics G: Nuclear and Particle Physics, 2011, 38 (2), pp.24005. 10.1088/0954-3899/38/2/024005. hal-00600873

\section{HAL Id: hal-00600873 https://hal.science/hal-00600873}

Submitted on 16 Jun 2011

HAL is a multi-disciplinary open access archive for the deposit and dissemination of scientific research documents, whether they are published or not. The documents may come from teaching and research institutions in France or abroad, or from public or private research centers.
L'archive ouverte pluridisciplinaire HAL, est destinée au dépôt et à la diffusion de documents scientifiques de niveau recherche, publiés ou non, émanant des établissements d'enseignement et de recherche français ou étrangers, des laboratoires publics ou privés. 


\title{
TOPICAL REVIEW
}

\section{Physics with REX-ISOLDE: from experiment to facility}

\author{
P Van Duppen ${ }^{1}$ and K Riisager ${ }^{2}$ \\ 1 Instituut voor Kern- en Stralingsfysica, Department of Physics and Astronomy, \\ K.U.Leuven, B-3001 Leuven \\ E-mail: piet.vanduppen@fys.kuleuven. be \\ 2 Department of Physics and Astronomy, University of Aarhus, DK-8000 Aarhus C \\ E-mail: kvr@phys.au.dk
}

\begin{abstract}
The REX-ISOLDE project and its physics program are presented. The innovative approach used to post-accelerate essentially all existing radioactive beams available at ISOLDE to $3 \mathrm{MeV} / \mathrm{u}$ is presented and beam properties are discussed. Isotopes as light as ${ }^{8} \mathrm{Li}$ and as heavy as ${ }^{224} \mathrm{Ra}$ have been used for Coulomb excitation, few-nucleon transfer reaction or fusion evaporation studies. Selected examples of the physics program, primarily utilizing the Miniball detector and segmented silicon detector arrays, are presented emphasizing some unique features of REX-ISOLDE. Finally, the HIE-ISOLDE project aiming at, amongst other goals, increasing the beam energy to $5.5 \mathrm{MeV} / \mathrm{u}$ and above, will be briefly discussed.
\end{abstract}

PACS numbers: 25.60.-t, 29.38.Gj

Submitted to: 


\section{Introduction and historical note}

The rich physics outcome of nuclear-reaction studies employing unstable targets and projectiles was foreseen already from the mid sixties [1], but although postacceleration at ISOLDE was considered as early as 1977 (and encouraged by Specht in 1981) [2], earnest considerations were only given from the end of the eighties through the PRIMA proposal [3]. The main aim of PRIMA was to study reactions of light nuclei of astrophysical relevance at energies up to about $1.4 \mathrm{MeV} / \mathrm{u}$, similar to what was being implemented at the time in Louvain-la-Neuve [4]. The rapid progress in the field quickly made PRIMA obsolete and led to discussions in larger circles, at first with a UK community around the ISIS facility at Rutherford Appelton Laboratory [5].

Traditionally, experiments at the ISOLDE facility have taken place at low-energy (up to $60 \mathrm{keV}$ ) via decay studies, ion (or atomic) beam measurements, ion manipulation e.g. in traps for mass measurements or implantation in host materials at temperatures from $10 \mathrm{mK}$ upwards for detailed studies of the decay process or studies of host properties in solid-state physics and life science. An overview of the many possibilities can be found from the laboratory portrait [6] published a decade ago. The strength of the science program at ISOLDE has for many years been based on the combination of the large number of different isotopes that can be produced with the many different techniques that can be employed to study them. It seemed natural to extend these techniques to include also reactions studies at moderate energies, such as Coulomb excitation, capture reactions and transfer reactions. The challenge was to devise an acceleration scheme that could take full advantage of the large range of isotopes available at ISOLDE.

The discussions in and around the ISOLDE community finally led to the REX-ISOLDE proposal in 1994 [7], the acronym coming from "Radioactive beam EXperiments at ISOLDE". Its key ingredient was an innovative charge breeding scheme combining bunching in a Penning trap with an electron beam ion source (EBIS), details will be given below. The philosophy was to separate as much as possible the production of singly charged radioactive ions from the subsequent ion manipulation to provide bunches of multiply charged ions needed for an efficient acceleration in all mass ranges. All components of REX-ISOLDE are therefore located inside the experimental hall which was helpful in the commissioning phase but places, at present, restrictions up on the intensity of some of the radioactive beams (RIB) to be accelerated. It was initiated as an experiment and the construction and initial running phase paid for by sources outside of CERN. The transfer to and integration into the CERN accelerator departments only took place after the final commissioning. The first physics runs were performed late 2001.

Detailed descriptions of the design of REX-ISOLDE, its commissioning and technical performance so far have been given elsewhere $[8,9,10,11,12,13,14]$. We shall focus on the main physics results achieved up to now and on the prospects for future experiments. The unique capabilities of REX-ISOLDE build on and are enhanced by the variety of ion manipulation techniques developed for and employed by the low-energy 
experiments at ISOLDE. To give a better perspective of the possibilities inherent in the REX-ISOLDE scheme we shall start by giving a brief overview of radioactive ion beam production and acceleration at ISOLDE. Section 3 outlines the present experimental instrumentation that determines which experiments can be undertaken. Section 4 presents a selection of physics results obtained so far in the more than 30 approved experiments. Finally, section 5 presents a brief outlook for the years ahead.

\section{Radioactive beam production at ISOLDE}

\subsection{The ISOLDE facility}

ISOLDE's radioactive ion beams are produced using the $1.4 \mathrm{GeV}$ proton beam from the PS-Booster accelerator at CERN. Beam pulses of about $310^{13}$ protons per pulse are send to the ISOLDE target every 1.2 seconds or multiples of this period resulting in an average beam intensity of 2 particle $\mu \mathrm{A}$. Various solid and liquid target materials are used to produce a wide spectrum of radioactive isotopes covering essentially the whole nuclear chart below uranium $(Z=92)$. Radioactive isotopes are produced via proton induced target fragmentation, spallation and fission reactions. The most frequently used target material is uranium carbide $\left(U C_{x}\right)$. In 2007, it was used for $67 \%$ of the beam time, followed by $\mathrm{SiC}(9 \%)$ and liquid lead $(6 \%)$. The radioactive products are thermalized in the target material and are subsequently transported from the target container to the ion source via diffusion and effusion processes. To speed up this process, the whole target ion source unit is kept at high temperature, typically $2000^{\circ} C$ for e.g. a $U C_{x}$ target. The speed of the diffusion and effusion process is governed by the physical and chemical properties of the radioactive elements of interest and of the specific materials that the target container, transfer line and ion source are made of. Consequently, radioactive isotopes that have small diffusion coefficients or very long lingering times at the surface of the target or container material, like the isotopes from some of the refractory elements, undergo radioactive decay before they are ionized and are thus lost. Once the atoms reach the ion source, a large fraction is ionized to a $1^{+}$charge state. Different ion sources are used at ISOLDE implementing different ionization mechanisms: electron impact ionization in high temperature plasma sources and, surface- and laser ionization in a hot cavity. The ions are subsequently extracted from the ion source, accelerated to typically $60 \mathrm{keV}$, mass separated according to mass-over-charge ratio using a dipole magnet and sent to the different experimental set-ups. Recently, an radiofrequency cooler-buncher has been installed in the low-energy beam line allowing delivery of short bunches of radioactive ion beams to experiments without a substantial loss in intensity. This development has resulted in a substantial increase in the peak-to-background ratio for e.g. the collinear laser spectroscopy measurements [15]. For a general description of the ISOLDE facility the reader is referred to [16]. A lay-out of the facility is shown in figure 1 .

ISOLDE's decades of experience in target-ion source development to make the whole 
process, from isotope production in the target to beam delivery to the experimental setup, efficient, fast and selective has resulted in a wide spectrum of beams. Radioactive beams with intensities reaching the $\mathrm{nA}$ level (e.g. ${ }^{213} \mathrm{Fr}$ with $8.410^{9}$ particles per second) and of short-lived isotopes with a few milliseconds half life (e.g. ${ }^{14} B e, T_{1 / 2}=4.45$ ms) have been achieved. These beams are used for experiments in the field of nuclear physics and weak interaction studies (taking $\sim 45 \%$ of the beam time), solid-state physics studies $(\sim 24 \%)$, atomic physics studies $(\sim 22 \%)$, particle and astrophysics studies $(\sim 10 \%)$ and, biology and medicine $(\sim 4 \%)$. Figure 2 overlays on the nuclear chart the current beams and the intensity available at $60 \mathrm{keV}$. The gaps with no beams available correspond to refractory-type elements (e.g. the $\mathrm{Ti}(Z=22)-\mathrm{V}(23)$, the $\operatorname{Zr}(40)$ - $\operatorname{Rh}(45)$ and the $\mathrm{Ta}(73)$ - $\operatorname{Pt}(78)$ regions) or extremely short-lived isotopes (e.g. the $N=128-130$ alpha emitting isotones with half-lives in the microsecond region). Next to the intensity is the purity of the final beam, an equally important parameter. Decades of RIB research has shown that different purification steps have to be applied sequentially as in most cases none of the purification steps result in a $100 \%$ pure beams. Different ways are implemented to purify ISOLDE's beams. The most straightforward way is to increase the wanted isotope over its isobaric contaminants by using the high-resolution mass separator [16]. Using the high-resolution mass separator, however, often induces losses in intensity. Other ways to purify the beam are making use of the physical and chemical properties of the element. For example, pure beams of gaseous elements are produced by cooling the transfer line between the target container and the ion source. In this way only the non-condensable elements (e.g. noble gases, gaseous molecules) are transferred to the ion source. Using surface ionization leads to beams of elements with a low ionization potential (alkaline-like or earth alkaline-like elements). The most elegant and effective way to purify the ion beam is the use of the so-called 'Resonant Ionization Laser Ion Source (RILIS)' [17, 18]. Resonant laser ionization is an element selective process whereby atoms are irradiated by two or three lasers exciting the electron in two or three resonant steps to the continuum. This allows the production of very pure beams as only atoms of the element of interest are ionized. Furthermore, because the nuclear spin determines the hyperfine structure of the atomic levels, isomeric beams can be produced. The RILIS is now used for more than half of the beam time and isomeric beams of isotopes from copper, silver, lead and polonium have been produced and used for experiments (see e.g. [19]). It should be noted that the pulse structure of the PS-Booster proton beam creates a particular time profile of the radioactive ion beam depending on a combination of the release properties and the half life of the isotope of interest [20]. The fact that this time profile can be very different for different isobaric isotopes is used to further purify the beam or to identify the experimental signals belonging to the isotopes under study (see e.g. [21]). 


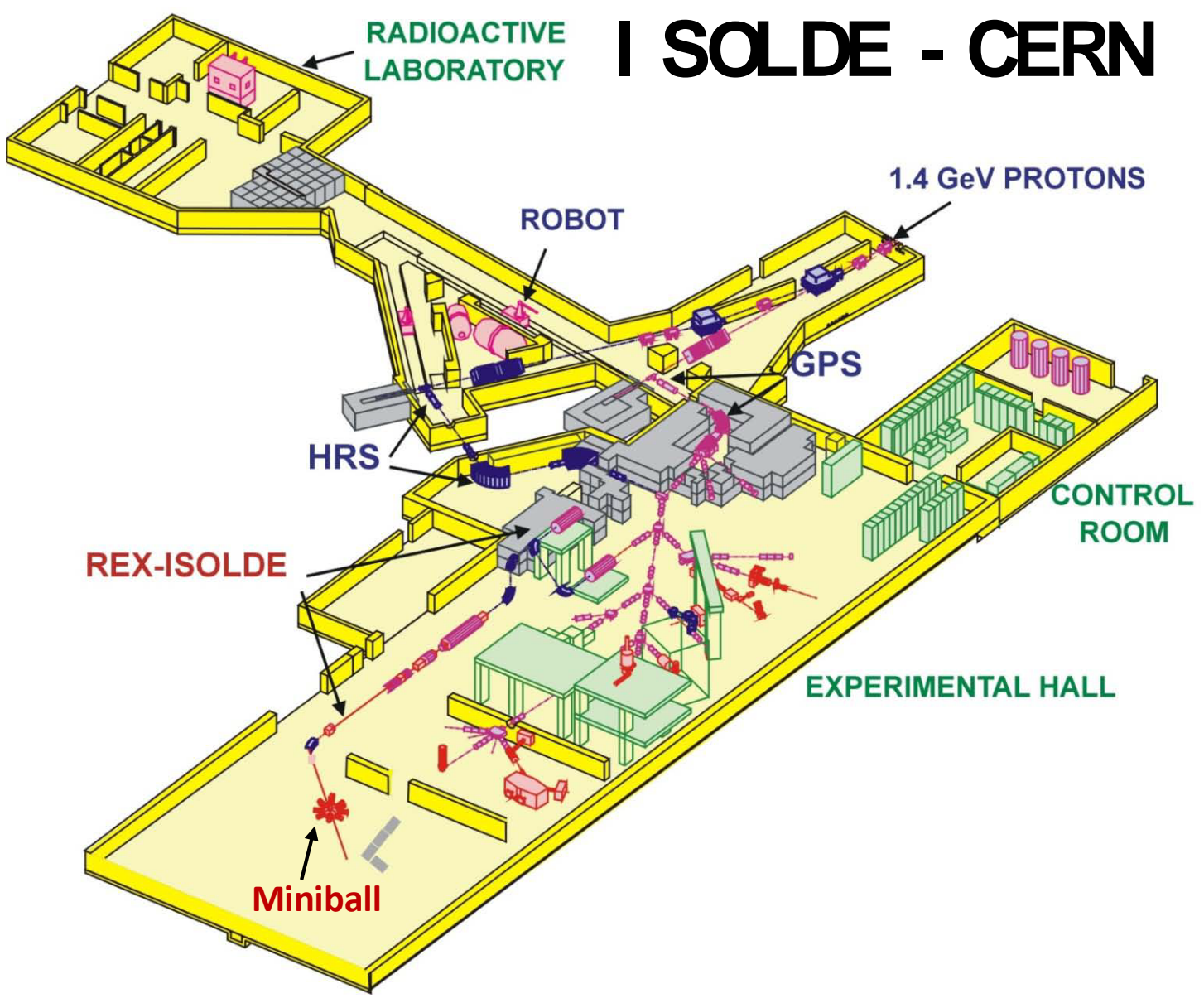

Figure 1. The lay-out of the ISOLDE facility is shown. The REX-ISOLDE accelerator complex can receive radioactive ion beam from both mass separators (HRS: high resolution separator, GPS: general purpose separator). Two beam ports are currently available for experiments with the energetic beams (colour online).

\subsection{The REX-ISOLDE project}

In order to boost the energy of the existing radioactive ion beams of ISOLDE from typically $60 \mathrm{keV}$ to a few $\mathrm{MeV} / \mathrm{u}$ a new concept was proposed whereby the existing singly-charged ion beams from ISOLDE could be accelerated in a universal, fast, efficient and cost-effective way $[7,13,14]$. The concept is based on ion beam cooling and bunching in the buffer gas of a Penning trap [23], charge-state breeding in an Electron Beam Ion Source (EBIS) [24] and post-acceleration in a room-temperature linear accelerator [25]. The original aim of the project was to prove the new concept's principle and to perform pilot experiments with short-lived post-accelerated radioactive ion beams [7]. The 60 $\mathrm{keV}$ radioactive ion beams from ISOLDE are retarded and continuously injected into a Penning trap situated at a high voltage platform with a potential of nearly $60 \mathrm{kV}$. In the trap the ions are accumulated and at the same time cooled via buffer gas collisions. After a fixed time, which is determined by the required charge breeding time, the ion bunch is extracted from the Penning trap, accelerated to $60 \mathrm{keV}$, transferred to the EBIS 


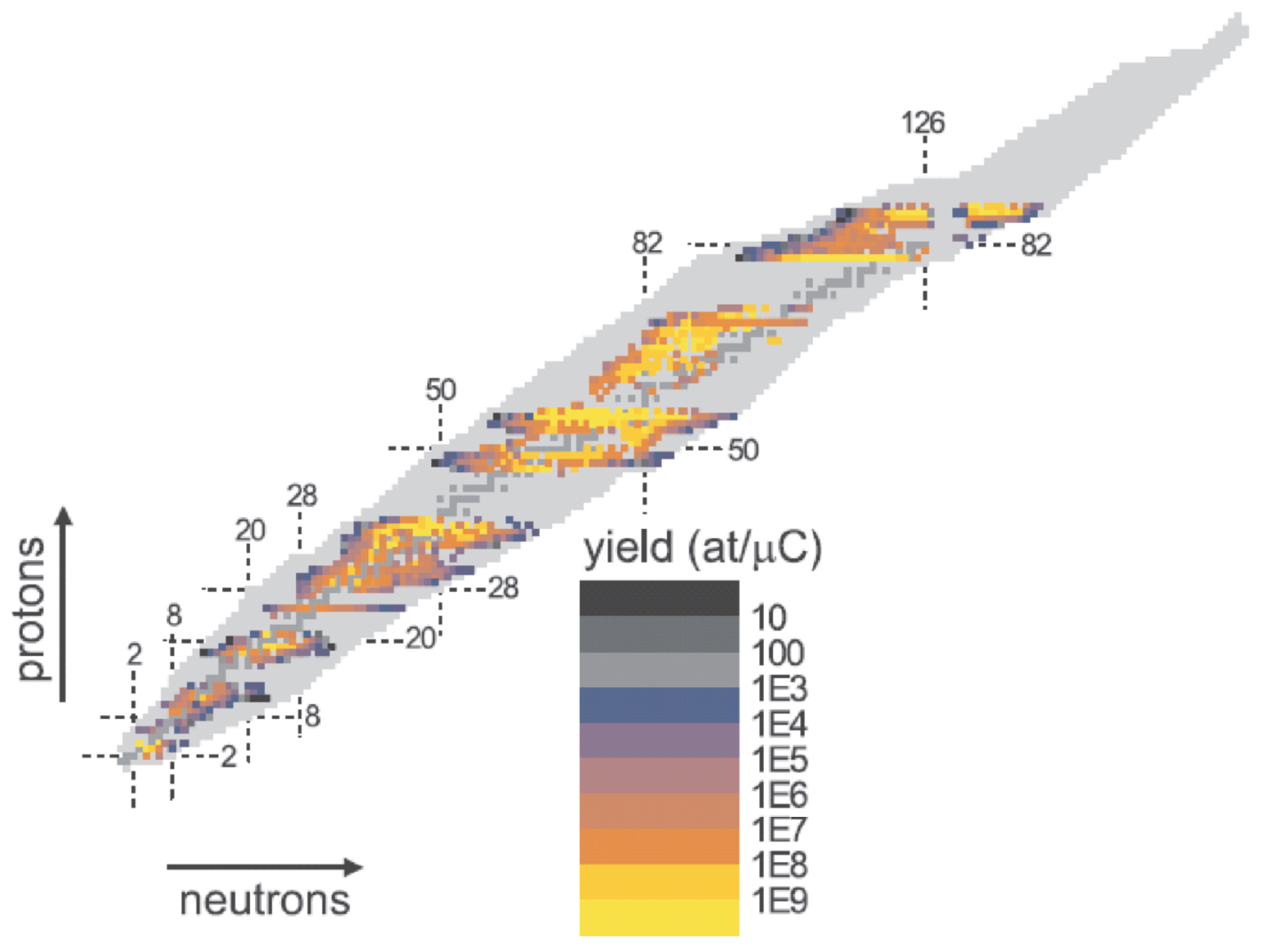

Figure 2. Isolde delivers beams that are used in an energy range from $10^{-6} \mathrm{eV}(10$ $\mathrm{mK}$ ) to $3 \mathrm{MeV} / \mathrm{u}$, with intensities up to $10^{10}$ ions per second, for isotopes with proton number from 2 to 88 and neutron number from 4 to 144; as light as ${ }^{6} \mathrm{He}$ to as heavy as ${ }^{232} R a$. Over 800 radioactive ion beams are available for experiments. The chart of nuclei is shown with a false color overlay of the radioactive beams available from ISOLDE. The color code gives the intensity of the $60 \mathrm{keV}$ beams. The post-accelerated beam intensity can be obtained by multiplying the latter values with the total REX efficiency that can be obtained from figure 3 [22] (colour online).

and retarded to ground potential prior to injection into the EBIS. The typical duration of the ion bunches extracted from the Penning trap is $10 \mu \mathrm{s}$. After charge breeding to the required mass-to-charge ratio $(\mathrm{A} / \mathrm{Q})<4.5$, the ion bunch is extracted from the EBIS, mass separated and injected in the radiofrequency quadrupole (RFQ) accelerator. The charge breeding time depends on the required charge-to-mass ratio and thus on the isotope of interest. It varies from typically $20 \mathrm{~ms}$ for light mass elements $(\mathrm{A}<40)$ to over $200 \mathrm{~ms}$ for the heavy elements in the lead region $(\mathrm{A} \simeq 200)$. The typical time width of the ion bunch extracted from the EBIS is $100 \mu \mathrm{s}$. As the required injection energy for the RFQ is $5 \mathrm{keV} / \mathrm{u}$, the EBIS platform potential is lowered from the $60 \mathrm{kV}$ retarding potential down to $\sim 20 \mathrm{kV}$ while charge breeding takes place. The room temperature accelerator typically runs with a $10 \%$ duty cycle (e.g. a radiofrequency-on period of $2 \mathrm{~ms}$ every $20 \mathrm{~ms}$ ). The RFQ accelerator is followed by an interdigital H-type (IH) structure with a final energy between 1.1 and $1.2 \mathrm{MeV} / \mathrm{u}$, three seven-gap resonators 
that create an energy range from 0.8 to $2.2 \mathrm{MeV} / \mathrm{u}$ and finally by a nine-gap resonator boosting the beam energy to a maximum of about $3 \mathrm{MeV} / \mathrm{u}$. The time structure of the accelerated ions is shown in figure 2 of [14]. It is determined by the repetition rate of the proton beam pulse from the PS-Booster (a proton pulse every $1.2 \mathrm{~s}$ or an integer multiple of this period), the release profile of the isotope of interest from the ISOLDE target ion source unit (typically tens of ms to s scale), the duty cycle of the accelerator (typically one $\sim 40$ to $150 \mu$ s wide pulse from the EBIS every $20 \mathrm{~ms}$ ) and the micro time structure of the linear accelerator (a few ns pulse width every $10 \mathrm{~ns}$ ).

\subsection{Specific RIB properties}

The universal principle behind REX-ISOLDE allows the acceleration literally all radioactive ion beams available at ISOLDE currently up to an energy of $3 \mathrm{MeV} / \mathrm{u}$. Beams as light as ${ }^{8} \mathrm{Li}$ and as heavy as ${ }^{224} \mathrm{Ra}$ have been accelerated with efficiencies varying from $\sim 2$ to $10 \%$ (see figure 3 ). These efficiency data were obtained in off- and on-line conditions and illustrate the potential of the new RIB acceleration concept. The total time it takes to cool, bunch, charge breed and accelerate depends on the isotope of interest and is mainly determined by the charge breeding time necessary to obtain a $\mathrm{A} / \mathrm{Q}$ ratio $<4.5$. It can be as fast as $12 \mathrm{~ms}$ in the case of ${ }^{9} \mathrm{Li}$ or up to about $400 \mathrm{~ms}$ for ${ }^{204} R n$ [13]. The purity of the accelerated beam depends on the production method of the isotope of interest, the target and ion source system used, the release time of the isotope of interest and of the contaminating isotopes and, again, the specific A/Q ratio used for the post-accelerated beam. Only by combining different selection principles based on physical, atomic and chemical properties, can the required purity be reached. A number of experiments have profited from these developments and below we list some typical cases.

In the case of Coulomb excitation studies of ${ }^{80} \mathrm{Zn}\left(\mathrm{T}_{1 / 2}=0.54 \mathrm{~s}\right)$, the suppression of the isobaric contamination was crucial (see sect. 4.2.2) [26, 27]. When using the RILIS to produce a beam of ${ }^{80} \mathrm{Zn}$, a strong isobaric contamination of ${ }^{80} R b$ and ${ }^{80} \mathrm{Ga}$ was observed. Rubidium and gallium have a low ionization potential and are swiftly surface ionized in the high temperature cavity. In order to reduce the intensity of these contaminants, two strategies were adopted: the use of a proton-to-neutron converter and a quartz transfer line between the target and the ion source. The low-energy neutrons produced by the converter mainly induce fission on ${ }^{238} U$ while spallation that produces ${ }^{80} R b$ is strongly suppressed [28] (see also figure 1 of [27]). Moreover the use of a quartz transfer line between the target container and the hot cavity prevents many elements to reach the ion source as they condense on the walls of the transfer line, except members of the chemical group 12 (zinc, cadmium, mercury). Thus while the transfer of ${ }^{80} \mathrm{Zn}$ from the target to the RILIS is hardly delayed, a strong delay is induced for gallium resulting in a strong suppression of ${ }^{80} G a\left(\mathrm{~T}_{1 / 2}=1.7 \mathrm{~s}\right)$ [29]. The next possible source of isobaric contamination in the accelerated beam stems from the EBIS. Beams from stable isotopes (like carbon, oxygen, nitrogen,..) are extracted from most ion sources. 
This can result in a strong isobaric contamination when the charge-to-mass ratio of the isotope of interest is similar to that of a stable isotope. However, because of its very clean vacuum conditions, an EBIS produces only relatively weak beams of stable isotopes and at several A/Q values virtually no stable beam is present. Finally, from the experimenters point of view, the use of laser ionization allows one to perform proper background measurements and to identify weak signals by simply switching the lasers on- and off at regular time intervals (see figures 6 and 8 of [27]).

Another example of using the ion beam manipulation capabilities of the REXISOLDE system to produce new beams is the production of neutron-rich iron beams $(\mathrm{Z}=26)$. Because of the chemical properties of iron, large delay losses are encountered in typical high temperature thick target systems. However radioactive ion beams from the neighboring element manganese $(Z=25)$ are obtained with high intensity and purity thanks to the laser ionization. As it takes a certain time to manipulate the ion bunches in the Penning trap and in the EBIS system, and the fact that this time may be varied, the short-lived manganese isotopes can decay inside the Penning trap and/or EBIS system resulting in beams of neutron-rich iron isotopes. This technique was successfully tested with injected beams of the ${ }^{61} \mathrm{Mn}\left(T_{1 / 2}=0.71 \mathrm{~s}\right)$ to produce beams of ${ }^{61} \mathrm{Fe}$ [30].

Since the time structure of the radioactive beam is dependent on the isotope of interest, analyzing the event time relative to the proton beam impact offers, in many cases, to possibility to further suppress unwanted contamination. This technique was used in the Coulomb excitation experiment of ${ }^{68} \mathrm{Ni}$ to reduce the strong isobaric contamination of ${ }^{68} \mathrm{Ga}$. The release of gallium isotopes is much faster compared to nickel therefore by setting proper beam gates or filtering the data in the off-line analysis the desired data can be obtained (see figure 1 of [21] and sect. 4.2.2).

A final example is the production of pure beams of ${ }^{70} \mathrm{Se}$ using a $\mathrm{ZrO} \mathrm{O}_{2}$ fiber target, again this beam can be overwhelmed by isobaric contaminants (see sect. 4.3) [31]. The key producing a pure beam ${ }^{70} \mathrm{Se}$ for this measurement was to extract the beam as a $1^{+}$ charged ${ }^{70} \mathrm{Se}^{12} \mathrm{C}^{16} \mathrm{O}$ molecule from the ISOLDE target-ion source system (in this case a high-temperature plasma ion source was used). This $\mathrm{A}=98$ beam, that is clean from any $\mathrm{A}=70$ contamination, is subsequently injected in the REX trap followed by the EBIS. In the latter the molecules are dissociated under the electron bombardment and ${ }^{70} \mathrm{Se}$ is further charge bred until the required $Q=19^{+}$for post-acceleration is reached. This resulted in an isobarically pure post-accelerated ${ }^{70} \mathrm{Se}$ beam.

\section{Instrumentation and experimental techniques}

The experimental program at REX-ISOLDE focusses on Coulomb excitation, fewnucleon transfer reaction studies and fusion evaporation studies using radioactive beams. Dedicated instrumentation for $\gamma$-ray and charge particle detection was developed especially tailored to the particularities of radioactive ion beam research: low beam intensities, low- $\gamma$-ray multiplicity and strong background radiation from the radioactivity of the beam itself. 


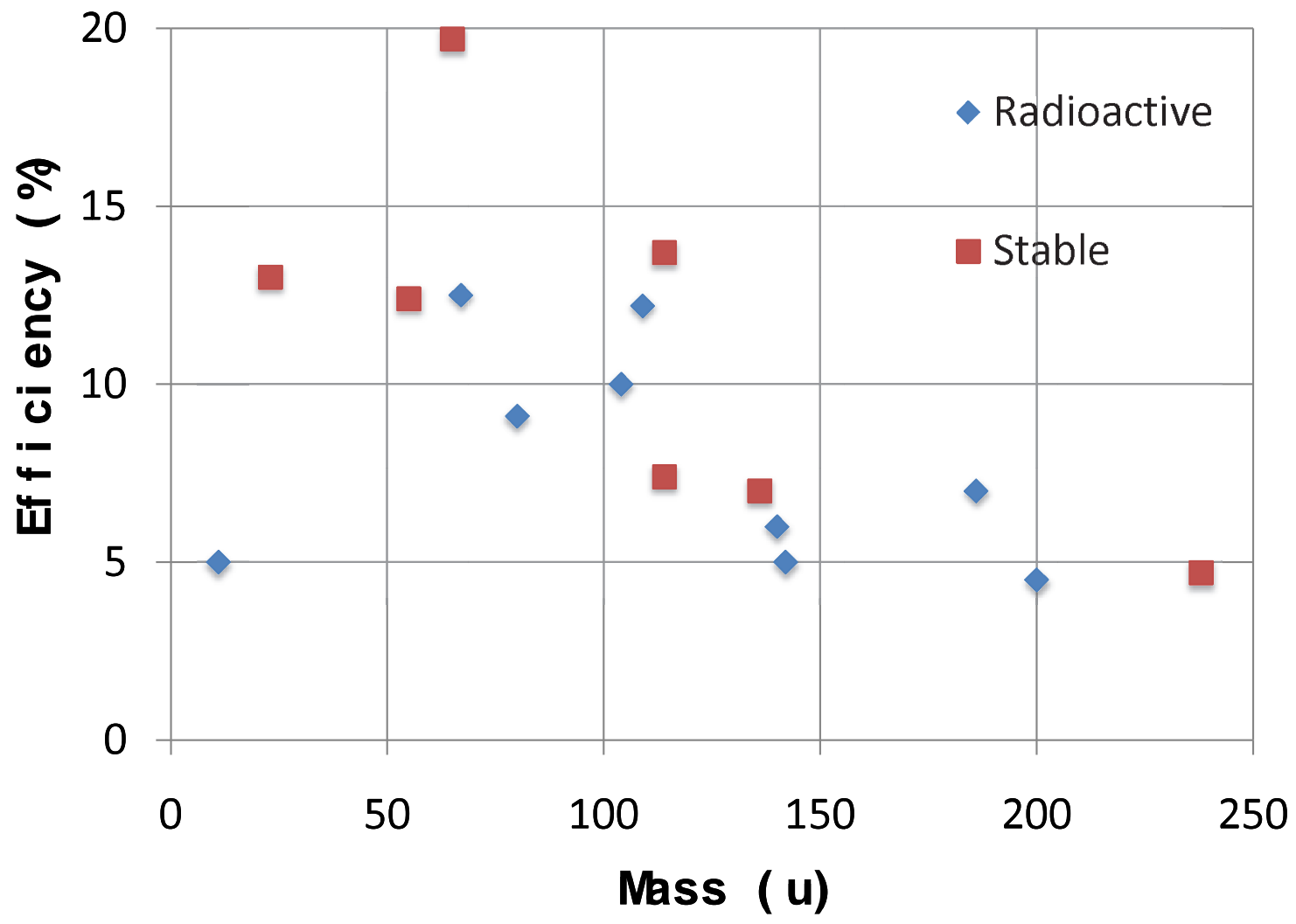

Figure 3. The product of the cooling, bunching and charge-state breeding efficiency is given as a function of mass. Values obtained for radioactive and stable isotopes are shown [13]. Tuning the REX bunching and charge-breeding system is easier with the higher intensity stable beams, therefore on average the efficiency values for stable beams are higher then the values obtained on-line for radioactive isotopes. The acceleration efficiency at maximum energy reaches $85 \%$. The original design values of the REX-ISOLDE project were an efficiency of $10 \%$ and $A<50$ [7] (colour online).

For $\gamma$-ray detection, an array of six-fold segmented, individually encapsulated and tapered germanium crystals was constructed [32]. The so-called Miniball array is optimized with respect to full-energy peak efficiency and angular resolution rather then resolving power. Miniball comprises eight cryostats, each containing three individual germanium crystals, which are mounted in close geometry around the target position. In its standard configuration for Coulomb excitation experiments, the typical target to detector distance is about $11 \mathrm{~cm}$ resulting in a solid-angle coverage of $\sim 60 \%$ of $4 \pi$. The photo-peak efficiency for the $1.3 \mathrm{MeV}$ reaches up to about $8 \%$. The 24 detectors routinely used with the Miniball array result in a granularity of 144 . By applying pulse shape analysis of the collected charges in each segment, 16 individual position can be distinguished in each segment [32]. This results in an optimum granularity above 2000 for the present Miniball array. The granularity is essential to obtain proper Doppler corrected spectra as the radioactive ion beams from ISOLDE have a typical velocity of $8.5 \%$ of the speed of light. However, with the present beam characteristics (typical diameter of $6 \mathrm{~mm}$ ) and the close proximity of the germanium detectors, the inclusion of 
the position determination using pulse shape analysis does not substantially improve the Doppler correction over the use of the electronic segmentation. In the case of Coulomb excitation experiments the spherical detection chamber is equipped with a double sided silicon strip detector (DSSSD) of the CD type [33] to detect projectile and/or target particles. The DSSSD allows determining the energy (velocity) and scattering angle of the detected particles, an essential ingredient for the Doppler correction of the $\gamma$-ray spectrum. It covers an angular range typically from $16^{\circ}$ to $53^{\circ}$ in the forward direction (laboratory frame), although this range can be varied by changing the target to detector distance.

To study few-nucleon transfer reactions using radioactive ion beams in inverse kinematics, two new detection set-ups were developed. The first, mainly used for scattering and transfer reactions with light nuclei $[34,35,36]$, consisted of one or more 40 to $60 \mu \mathrm{m}$ thick 16 by 16 double-sided Si strip detectors with a specially developed [37] thin dead layer, backed by 1000 to $1500 \mu \mathrm{m}$ thick Si pad detectors. This setup is typically adapted from run to run, one implementation is shown in figure 4 that also displays the second set-up, called T-REX (the T stands for transfer), mainly used for transfer reactions using heavier mass beams and combining particle detection with gamma detection $[38,39]$. It consists of the Miniball array to detect $\gamma$-rays in coincidence with particles detected by an array of segmented Si detectors. The array of silicon detectors exhibits a nearly $4 \pi$ angular coverage. The array comprises two CD detectors, in the forward and the backward direction, and a barrel of eight planar DSSSD's around $90^{\circ}$. The forward CD detector consists of two layers and is used as a $\triangle E$-E telescope. The four forward detectors of the barrel are also $\triangle E$-E-telescopes. The $\triangle$ E-detector (thickness $140 \mu \mathrm{m}$ ) is segmented in 16 strips perpendicular to the beam axis and positional information along the stripes is obtained from the charge division on a resistive layer. The E-detector is not segmented and has a thickness of $1000 \mu \mathrm{m}$. The four backward detectors are thick $(500 \mu \mathrm{m})$, segmented E-detectors. The use of $\triangle E$-E-telescopes in forward direction enables the identification of light particles $(\mathrm{p}, \mathrm{d}, \mathrm{t}, \alpha)$.

\section{Physics program}

\subsection{Light nuclei}

The light nuclei is where we have the most intimate knowledge of the nuclear driplines [40] and it is therefore the natural starting point for investigations of nuclei as open quantum systems [41]. Among the phenomena seen here are nuclear halos [42], nuclear clusters and molecules [43] and changes in magic numbers [44]. Precision reaction experiments performed at a few $\mathrm{MeV} / \mathrm{u}$ - such as elastic scattering, few nucleon transfer and fusion experiments - will give valuable information on the structure of light exotic nuclei, but one important aspect is that the dynamics of the reactions may change due to continuum effects. This adds to the richness of the physics in this region and in some 


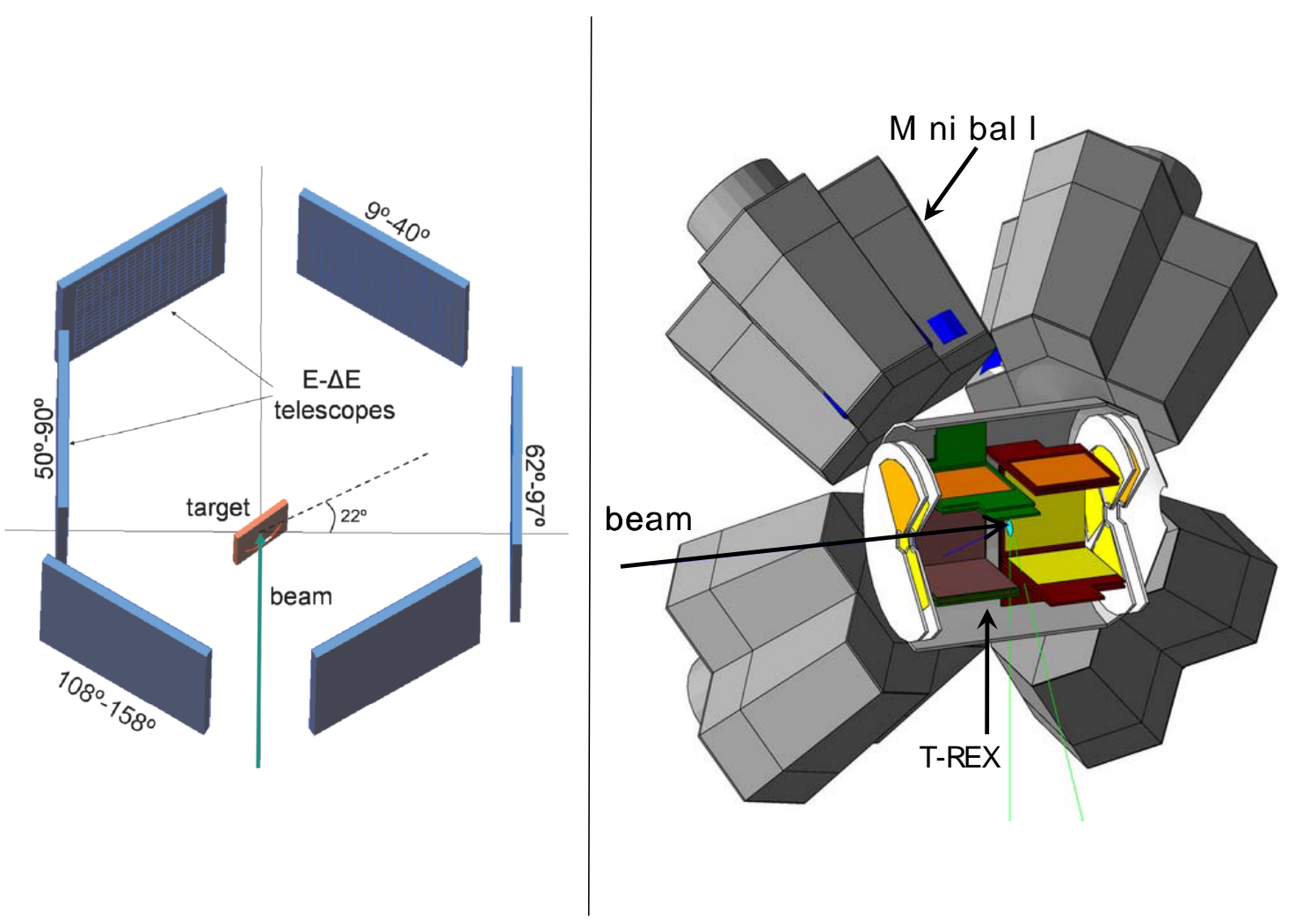

Figure 4. Left: the set-up used in the ${ }^{11}$ Be scattering experiments [36, 61] mentioned in section 4.1. Right: The T-REX silicon barrel detector used in combination with the Miniball germanium array for few-nucleon transfer reaction experiments using the radioactive ion beams from ISOLDE mentioned in section 4.1, 4.2.1 and 4.2.2 (from $[38,39])$ (colour online).

respects still presents a challenge for the theoretical modeling.

Most of the interesting reaction channels will contain nuclei with quite low level densities so that the resolution obtainable via detection of the outgoing particles often suffices to uniquely identify the excited state of the populated nucleus. Gamma-ray detection is therefore not always needed and several experiments have taken place without the Miniball array in an optimized set-up.

A series of experiments with ${ }^{9} \mathrm{Li}$ were among the first initiated at REX-ISOLDE. The first runs $[34,45,46]$ were performed at the original energy of $2.36 \mathrm{MeV} / \mathrm{u}$, after the addition of the 9-gap resonator an energy of $2.77 \mathrm{MeV} / \mathrm{u}$ could be achieved. The nucleus ${ }^{9} \mathrm{Li}$ is the most neutron-rich $A=9$ nucleus, it has a neutron separation energy of 4.064 MeV [47] and has one excited state at 2.69 MeV [48]. The main aim of the experiment was to acquire through the $\mathrm{d}\left({ }^{9} \mathrm{Li}, \mathrm{p}\right){ }^{10} \mathrm{Li}$ reaction more detailed information on the structure of the unbound system ${ }^{10} \mathrm{Li}$, required in order to obtain a coherent and complete description of the halo nucleus ${ }^{11} \mathrm{Li}$. Several earlier experiments gave indications for the presence of both low-lying s- and p-wave states in the ${ }^{9} \mathrm{Li}+\mathrm{n}$ system, see $[40,42]$ and references therein. The REX experiment confirmed this [46] through a simultaneous analysis of the energy spectrum and angular distribution within the 

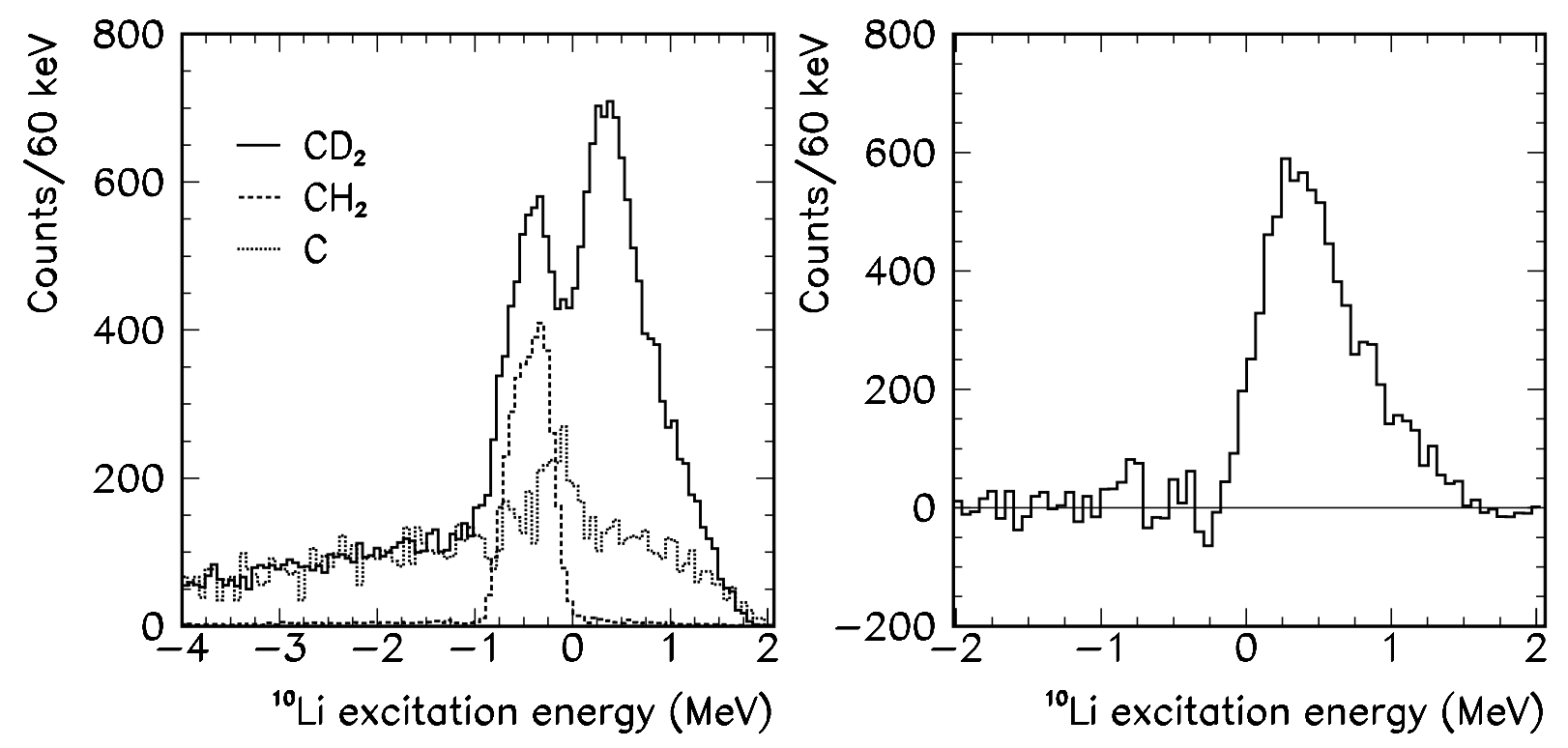

Figure 5. Countrate of the $\mathrm{d}\left({ }^{9} \mathrm{Li}, \mathrm{p}\right){ }^{10} \mathrm{Li}$ reaction at $2.77 \mathrm{MeV} / \mathrm{u}[49]$ is shown as a function of the excitation energy of ${ }^{10} \mathrm{Li}$. The left panel shows the spectrum recorded from a $\mathrm{CD}_{2}$ target as well as (normalized) background spectra from $\mathrm{CH}_{2}$ and pure $\mathrm{C}$ targets. The right panel gives the resulting final distribution.

Coupled-Channels Born approximation (CCBA) formalism adapted for unbound final states. In this way all continuum-continuum couplings could be included. The p-state was found at $0.38 \mathrm{MeV}$ whereas the s-state appeared as a virtual state with a negative scattering length 13-24 fm. Figure 5 shows the energy distribution of ${ }^{10} \mathrm{Li}$ with improved statistics from the most recent run [49] that still is under analysis. Since a $\mathrm{CD}_{2}$ target with a few percent contaminant of $\mathrm{CH}_{2}$ was used, separate runs on a pure $\mathrm{C}$ target and on a $\mathrm{CH}_{2}$ target were made in order to control the background reactions.

The scattering in the $d+{ }^{9} \mathrm{Li}$ system itself is actually also of interest since this is the final state in the beta-delayed deuteron decay of ${ }^{11} \mathrm{Li}$ where the decays apparently proceed directly into the continuum. The information from reaction experiments will therefore be crucial for constraining the interpretation of the decay experiment [50].

Among the other physics questions addressed by this experiment is the question of spectroscopic factors in light nuclei. The nuclei are so light that predictions can be made from ab initio calculations, but the ${ }^{9} \mathrm{Li}(\mathrm{d}, \mathrm{t})^{8} \mathrm{Li}$ data gave spectroscopic factors twice as large as predicted by theory [45] which could be due to the low reaction energy or to the fact that we are dealing with somewhat loosely bound systems. However, the same reaction done at ISAC at $1.68 \mathrm{MeV} / \mathrm{u}$ reported no problems [51] and seemed consistent with several ${ }^{8} \mathrm{Li}(\mathrm{d}, \mathrm{p})$ results below $10 \mathrm{MeV} / \mathrm{u}$, see e.g. [52]. This is an important point to settle since knock-out reactions at higher energy, e.g. at MSU [53], gives a spectroscopic strength that varies systematically with the difference in separation energy between protons and neutrons. This must be checked [54] via low energy transfer reactions that are a very well understood probe closer to stability, one example being the systematic study of $(\mathrm{d}, \mathrm{p})$ and $(\mathrm{p}, \mathrm{d})$ reactions in ground-state to ground-state transitions [55], but 


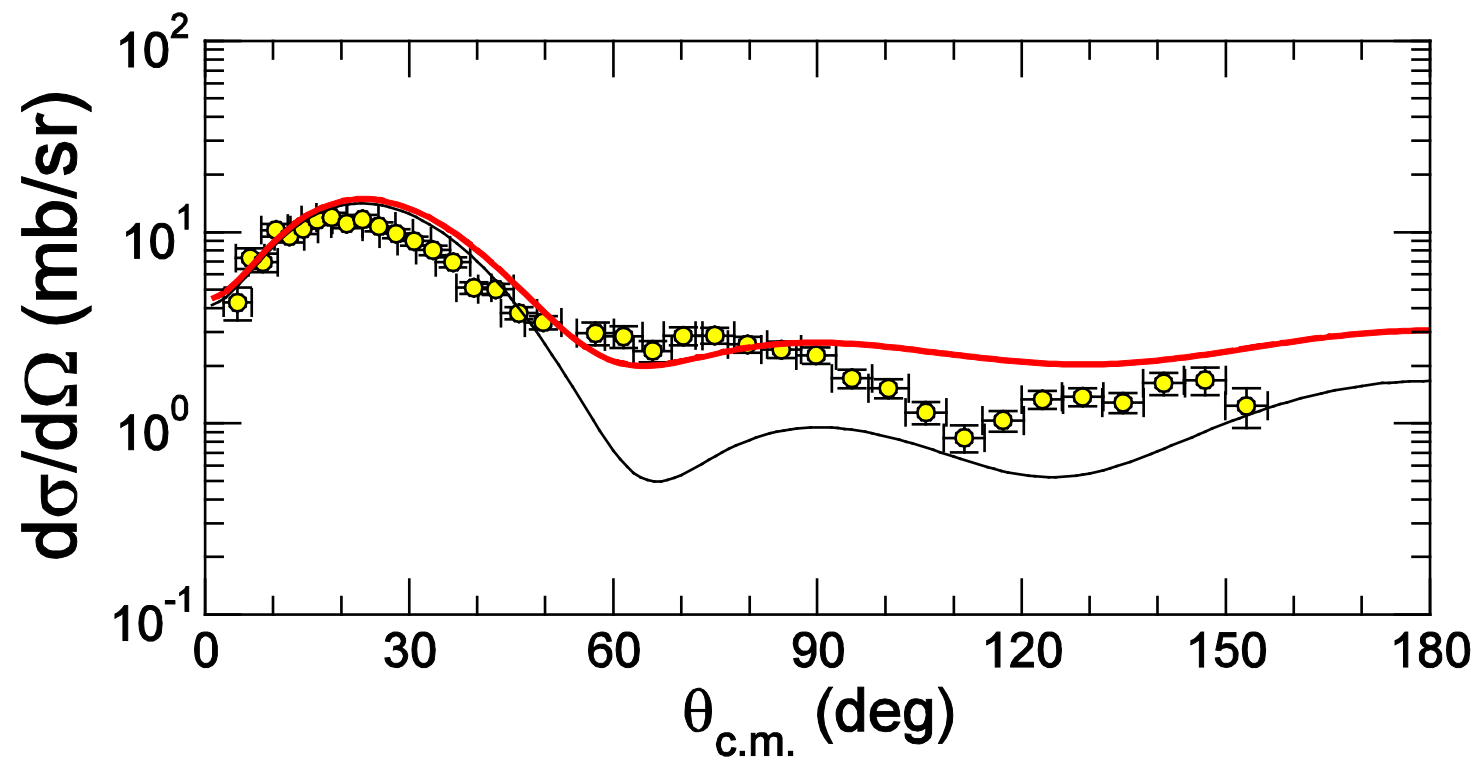

Figure 6. The differential cross-section for the $d\left({ }^{8} \mathrm{Li}, \mathrm{p}\right){ }^{9} \mathrm{Li}(\mathrm{gs})$ transition at 3.15 $\mathrm{MeV} / \mathrm{u}$ is shown versus the angle in the centre-of-mass system [35]. The lower curve is a DWBA calculation of the reaction and the upper curve a CCBA calculation, see the text (colour online).

it is important to note that the physical meaning of spectroscopic factors is still being discussed theoretically [56]. To see how transitions between the ${ }^{8} \mathrm{Li}$ and ${ }^{9} \mathrm{Li}$ nuclei behave will be an important check, but needs careful experiments that are analyzed in a consistent manner. First data have already been recorded at REX-ISOLDE with a ${ }^{8} \mathrm{Li}$ beam as shown in figure 6 . The final theoretical analysis is being done presently, but it is already now clear that a standard Distorted Wave Born Approximation (DWBA) is not sufficient to describe even the transition between the reasonably well bound ground states of ${ }^{8} \mathrm{Li}$ and ${ }^{9} \mathrm{Li}$ when one goes beyond the first maximum in the differential cross section. A significantly better job is done by employing the Coupled-Channels Born Approximation, i.e. it is crucial to get continuum effects under control before any physics conclusions can be drawn. Even the position of the first minimum is off in DWBA that only captures the very basic features of the reaction. This reinforces the need to cover an extended angular range experimentally such that a more complete picture of the reaction can be obtained.

An intense beam of the halo nucleus ${ }^{11} \mathrm{Be}$ was developed at ISOLDE about a decade ago in order to enable precision measurements of its magnetic moment [57]. Several beta-decay experiments also benefitted from this development and it was logical to also undertake reaction measurements at low energy to complement and further elucidate the many results obtained earlier at higher beam energies for this prototype one-neutron halo. Transfer reactions can elucidate the structural changes between ${ }^{11} \mathrm{Be}$ and its neighbors ${ }^{10,12} \mathrm{Be}$ as shown by the $\mathrm{p}\left({ }^{11} \mathrm{Be},{ }^{10} \mathrm{Be}\right) \mathrm{d}$ reaction performed at $35.3 \mathrm{MeV} / \mathrm{u}$ at GANIL [58] through which the amount of core excitation in the ${ }^{11}$ Be ground state was probed or the recent ${ }^{11} \mathrm{Be}(\mathrm{d}, \mathrm{p})$ reactions performed at $5 \mathrm{MeV} / \mathrm{u}$ at TRIUMF [59] where 
spectroscopic factors was extracted for states in ${ }^{12} \mathrm{Be}$. A more extensive study of ${ }^{12} \mathrm{Be}$ through ${ }^{11} \mathrm{Be}+\mathrm{d}$ reactions at $2-3 \mathrm{MeV} / \mathrm{u}$ has been initiated at REX-ISOLDE which very recently also included detection of gamma rays in the Miniball array.

The dynamics of reactions of halo nuclei with intermediate to heavy mass nuclei at energies close to the Coulomb barrier continues to present challenges [60]. Following initial experiments with the two-neutron halo nucleus ${ }^{6} \mathrm{He}$ two experiments at REXISOLDE have contributed by investigating reactions on the one-neutron halo nucleus ${ }^{11} \mathrm{Be}$ (hoped to be simpler to treat theoretically) on ${ }^{64} \mathrm{Zn}[61]$ and ${ }^{120} \mathrm{Sn}$ [36]. In the former case data were also recorded for ${ }^{9} \mathrm{Be}$ and ${ }^{10} \mathrm{Be}$ beams (at LNS-Catania and REXISOLDE, respectively) thereby allowing the better isolation of features unique to ${ }^{11} \mathrm{Be}$. Whereas the Coulomb-nuclear interference peak, often referred to as the rainbow, is prominent in reactions with stable beams the data show a clear suppression for ${ }^{11} \mathrm{Be}$ that furthermore sets in at smaller scattering angles. One way of understanding this feature is through enhanced coupling to low-lying continuum states. The enhanced role of break-up processes seen in earlier experiments at high energy therefore continues down to the Coulomb barrier.

\subsection{Shell evolution}

The nuclear shell model is very successful in describing the structure of atomic nuclei situated around closed proton and/or neutron shells. The availability of beams of exotic nuclei allows one to test particular aspects of the nuclear shell model very far from stability: evolution of single-particle states, the disappearance and appearance of magic numbers and the validity of new effective interactions. Important progress in largescale shell model calculations has been made in recent years [62, 63], new interactions have been proposed for the heavier fpg-shell region and especially the role of central and tensor forces has been highlighted [64,65]. The region of the island of inversion around the $\mathrm{N}=20$ closed neutron shell, the neutron-rich nickel region $(Z=28)$ and the neutron-deficient and neutron-rich tin region $(Z=50)$ has been investigated at ISOLDE using Coulomb excitation and few-nucleon transfer reactions.

4.2.1. The $N=20$ region The island of inversion around ${ }^{32} M g(\mathrm{~N}=20)$ has been intensively studied using complementary methods which resulted in a wealth of data. Beta-decay studies, laser spectroscopy measurements and mass measurements all indicated unusual properties for the ground-state and low-lying excited states of the neutron deficient isotopes around ${ }^{32} \mathrm{Mg}$ (see e.g. [44, 66]. The availability of energetic radioactive ion beams of ${ }^{32} \mathrm{Mg}$ and nuclei in its neighborhood allowed the study of this phenomena in more detail and further in-beam spectroscopy (see e.g. [67]), Coulomb excitation (see e.g. [68]) and transfer reaction studies (see e.g. [69]) were performed. The combination of these experimental data and new theoretical calculations (see e.g. $[70,71])$ delineated the so-called island of inversion and proposed as a driving mechanism to explain these observations multi particle - multi hole $(p-h)$ excitations across the $N=20$ 
shell. This hypothesis gives rise to specific properties that can be probed using different experimental techniques: i) particle-hole excitations through a closed shell configuration result in an increased collectivity as it enhances the number of active nucleons and thus the number of deformation driving proton-neutron interactions; ii) spin and parities, and the single-particle nature of some low-lying states should reflect excitation across the $\mathrm{N}=20$ shell and iii) a $0^{+}$state presumably of a neutron two-particle two-hole $(2 \mathrm{p}-2 \mathrm{~h})$ nature should co-exist with the ground-state configurations or could even become the ground state. A sensitive probe to study collective properties is Coulomb excitation by which we may deduce transition or diagonal matrix elements between or of excited states. Single particle properties of the low-lying states can be probed using one-neutron transfer reactions while the existence of excited $0^{+}$states and the study of their $2 \mathrm{p}-$ $2 \mathrm{~h}$ character can be probed using two-neutron transfer reactions of the type $(\mathrm{t}, \mathrm{p})$. At REX-ISOLDE Coulomb excitation experiments using low-energy beams of ${ }^{30,32} \mathrm{Mg}$ were performed $[72,75]$. The $\gamma$-ray spectra from which the $B\left(E 2: 0^{+}-2^{+}\right)$result of ${ }^{32} \mathrm{Mg}$ $\left(T_{1 / 2}=95 \mathrm{~ms}\right)$ were extracted are shown in figure 7 . The final result is currently being prepared for publication [73]. By comparing the intensity in the $\gamma$ rays observed in the top and bottom spectrum the $B\left(E 2: 0^{+}-2^{+}\right)$for ${ }^{32} M g$ can be extracted based on the known transition matrix elements of the $\gamma$-ray observed in ${ }^{107} \mathrm{Ag}$. In this manner the result does not depend on the absolute radioactive beam intensity, the target thickness or the absolute $\gamma$ - and particle detection efficiency. Only the relative $\gamma$-ray efficiency and the purity of the radioactive ion beam must be determined. The results firmly establish an increase in collectivity in ${ }^{32} \mathrm{Mg}$ when moving from ${ }^{30} \mathrm{Mg}$, that exhibits a low degree of collectivity, to the $\mathrm{N}=20$ neutron shell closure. Essential for this and other measurements at REX-ISOLDE was not only sufficient beam intensity but, sometimes more importantly, the high degree of beam purity and the possibility to measure the beam purity accurately using proper detectors or simply using the possibility switching the lasers on and off during the experiment. Apart from intensive Coulomb excitation measurements, one-neutron and two-neutron transfer reactions have been performed on ${ }^{30} \mathrm{Mg}$. From the first $d\left({ }^{30} \mathrm{Mg}, p\right)^{31} \mathrm{Mg}$ experiment using a set-up with only a limited angular coverage for the proton detection no definite conclusions could be drawn [74]. With the new T-REX set-up, $d\left({ }^{30} M g, p\right)^{31} M g$ was repeated and the two-neutron transfer on ${ }^{30} M g$ was studied using a radioactive tritium target $\left(t\left({ }^{30} M g, p\right){ }^{32} M g\right)[39,76]$. The tritium target was a thin strip of $500 \mu \mathrm{g} / \mathrm{cm}^{2}$ thick metallic Ti foil loaded with a atomic ratio ${ }^{3} \mathrm{H} / \mathrm{Ti}$ of 1.5 corresponding to a target thickness of $40 \mu \mathrm{g} / \mathrm{cm}^{2}$ of tritium. Typical beam intensities of a few times $10^{4}$ particles per second were used. The two-neutron transfer experiment clearly revealed the existence of an excited $0^{+}$state in ${ }^{32} \mathrm{Mg}$ at an excitation energy of $1058 \mathrm{keV}$. Figure 8 shows the proton angular distribution obtained for the ground state and the newly identified excited state. From the angular distribution it can be concluded that this state is a $0^{+}$state only $178 \mathrm{keV}$ above the known $2^{+}$state in ${ }^{32} \mathrm{Mg}$. From the experimental cross section of the ground state population, it could be concluded that the $\nu 2 \mathrm{p} 3 / 2$ contribution as deduced from Monte-Carlo shell-model calculations is underestimated, while the cross section to populate the excited $0^{+}$state 


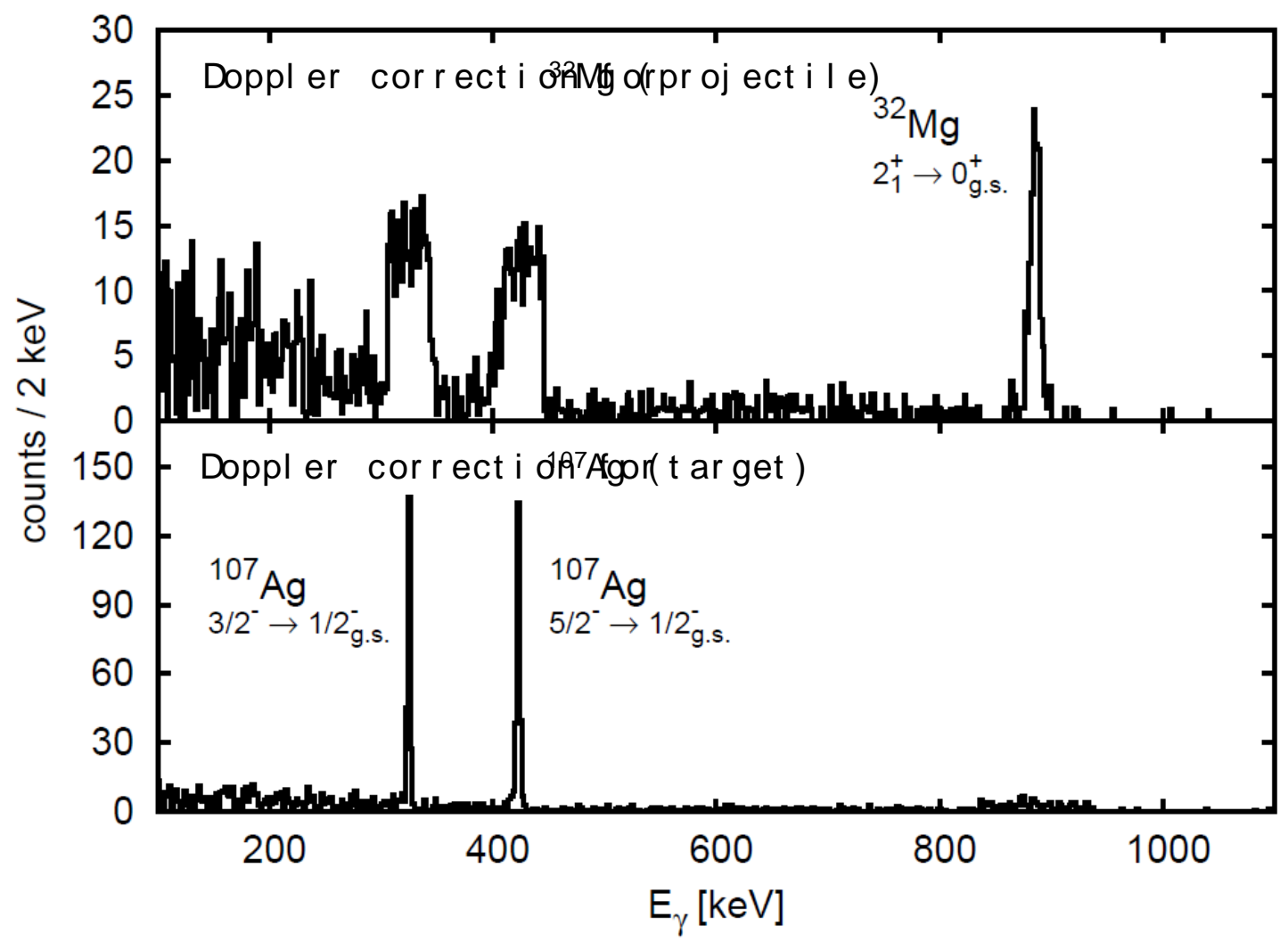

Figure 7. $\gamma$-ray spectra obtained after Coulomb excitation of a ${ }^{32} \mathrm{Mg}$ beam at 2.84 $\mathrm{MeV} / \mathrm{u}$ on a $4.4 \mathrm{mg} / \mathrm{cm}^{2}$ thick ${ }^{107} \mathrm{Ag}$ target are shown after proper Doppler correction for the projectile (top) and for the target nuclei (bottom). The known transitions in ${ }^{107} \mathrm{Ag}$ and the $2^{+}-0^{+}$in ${ }^{32} \mathrm{Mg}$ are clearly identified. Note the very low background conditions (adapted from [75])

can be fairly well described within a shell-model interpretation assuming rather pure $s d$ configurations [76].

4.2.2. Neutron-rich region around $Z=28$ The experimentally observed nickel $(\mathrm{Z}=28)$ isotopes span a very wide range including a number of doubly closed shell nuclei from ${ }^{48} \mathrm{Ni}$, where two-proton ground state decay was observed [77], over ${ }^{56} \mathrm{Ni}$, where enhanced core polarization has been observed $[78,79]$ towards ${ }^{78} \mathrm{Ni}$, a nucleus of particular interest for nuclear-structure and nuclear astrophysics [80]. This region of the nuclear chart thus represents an ideal testing ground for large-scale shell model calculations and the new interactions used. In addition ${ }^{68} \mathrm{Ni}$, with its $\mathrm{N}=40$ sub-shell closure is an interesting case as the importance of this shell closure, a parity changing harmonic oscillator closure, is still debated. The first excited state of ${ }^{68} \mathrm{Ni}$ is a $0^{+}$state and the first $2^{+}$state is located at a high excitation energy while the $B\left(E 2: 0^{+}-2^{+}\right)$value appears to be low compared to the neighboring nuclei. Although this might be interpreted as a signature for a strong shell closure, it has recently been suggested that the parity change between the $\nu p_{1 / 2}$ and $\nu g_{9 / 2}$ neutron orbitals and the small angular momentum of the former are 

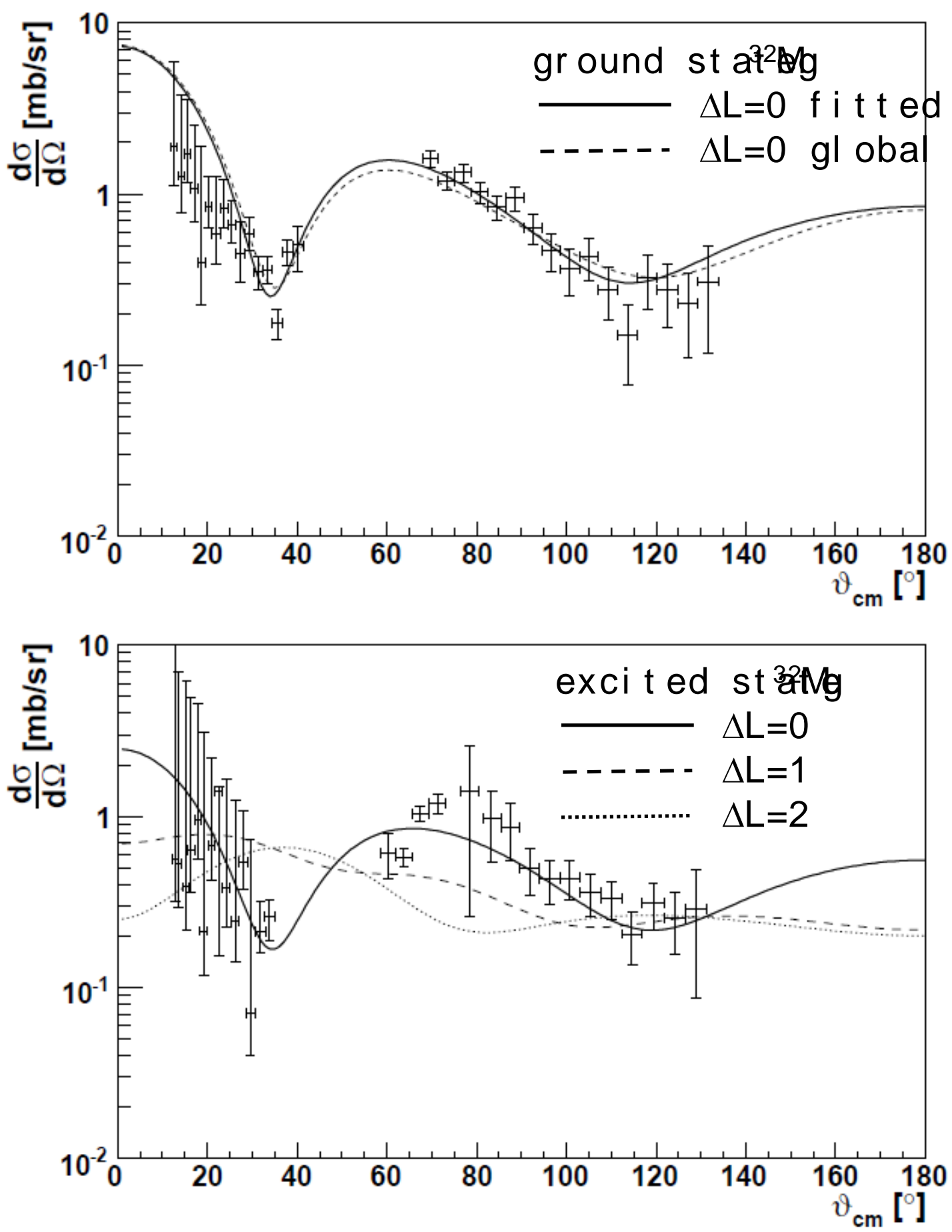

Figure 8. Proton angular distributions for the population of the ground state (top) and excited state at $1058 \mathrm{keV}$ (bottom) in the ${ }^{30} \mathrm{Mg}(t, p)^{32} \mathrm{Mg}$ reaction. The curves in the top panel are from DWBA calculations using fitted (solid) and global (dashed) optical model parameters. The curves in the bottom panel include DWBA calculations for $\Delta \mathrm{L}=0,1$ and 2 transfer. The excited state is populated with $\Delta \mathrm{L}=0$ transfer leading to the identification of a $0_{2}^{+}$in ${ }^{32} \mathrm{Mg}$ (adapted from [76]). 
the cause of the observed phenomena [44]. The structure of the neighboring nuclei of ${ }^{68} \mathrm{Ni}$ (coupling one proton or one neutron particle or hole) studied in beta decay can be reasonably well described as core-coupled states but indicate at the same time the importance of neutron excitations through $\mathrm{N}=40$ and proton excitations through $\mathrm{Z}=28$ (see e.g. [81]). The importance of proton excitations through the $\mathrm{Z}=28$ closed shell is e.g. nicely demonstrated by the recent observation of a low-lying long-lived $\left(1 / 2^{-}\right)$ isomer in ${ }^{67} \mathrm{Co}$ ([82], recently confirmed in [83]); taking away only one proton from ${ }^{68} \mathrm{Ni}$ already induces the obliteration of the $\mathrm{N}=40$ sub-shell gap and sets in a region of deformation below $\mathrm{Z}=28$.

In order to obtain a better understanding of the evolution of the nuclear structure between $\mathrm{N}=40$ and $\mathrm{N}=50$, Coulomb excitation experiments were performed using postaccelerated beams of ${ }^{68} \mathrm{Ni}$ [21], of the ground state and the long-lived isomers in ${ }^{68,70} \mathrm{Cu}$ [84], of the neutron-rich odd-mass copper isotopes $\left({ }^{67-73} \mathrm{Cu}\right)$ [85], of the neutron-rich zinc isotopes up to ${ }^{80} \mathrm{Zn}$ at the closed $\mathrm{N}=50$ neutron shell $[26,27]$ and of the neutronrich manganese and iron beams [30]. The ${ }^{68} \mathrm{Ni}$ experiment confirmed the low value for the $B\left(E 2: 0^{+}-2^{+}\right)$that was previously deduced from intermediate-energy Coulomb excitation [86] and the study of the ${ }^{68,70} \mathrm{Cu}$ isomers confirmed the core-coupled character of the states probed in the experiments and indicated only weak polarization effects induced by the extra-proton and neutron coupled to the ${ }^{68} \mathrm{Ni}$. However, including data from ${ }^{72} \mathrm{Cu}$ shows that the coupling of at least two like quasi-particles to ${ }^{68} \mathrm{Ni}$ weakens the stabilization effects of the $\mathrm{N}=40$ sub-shell and $\mathrm{Z}=28$ shell gaps [84]. These phenomena were further explored in the Coulomb excitation study of the odd-mass copper isotopes and revealed, at low excitation energy, a clear interplay between single-particle and collective effects in the odd-A copper isotopes beyond $\mathrm{N}=40$. These isotopes were, prior to the Coulomb excitation experiments, characterized by a $3 / 2^{-}$ground state and a lowlying $5 / 2^{-}$state whose excitation energy decreased dramatically beyond $\mathrm{N}=40$ when moving towards $\mathrm{N}=50$ [87]. The low $\mathrm{B}(\mathrm{E} 2)$ value between these two states confirmed their single-particle nature. But surprisingly, strong excitation was observed towards a low-lying $1 / 2^{-}$state, that followed the excitation energy behavior of the $5 / 2^{-}$state very closely, indicating a strong enhancement of collectivity in these neutron-rich copper isotopes as shown in figure 9 [85]. The experiment also allowed the identification of the $\left(7 / 2^{-}\right)$core-coupled state as its energy and B(E2) value are very similar to those of the $2^{+}$state in the neighboring even-even nickel isotopes. In these series of neutron-rich copper isotopes, two $\left(7 / 2^{-}\right)$states were identified from previous studies. The fact that only one of them is populated in Coulomb excitation points to a single-particle character for the other one; suggesting it to be a plausible candidate for a proton intruder state based on a $\pi(1 \mathrm{p}-2 \mathrm{~h})$ excitation across $\mathrm{Z}=28$.

Recent large-scale shell-model calculations performed with a new interaction for the fpg-shell nuclei in a valence space defined around the ${ }^{56} \mathrm{Ni}$ core were able to reproduce the trend of the $5 / 2^{-}$state excitation energy, however they failed to reproduce the properties of the $1 / 2^{-}$state (see figure 13 from [62]). Another recent calculation performed in a valence space outside the ${ }^{48} \mathrm{Ca}$ core and including adjustments to 


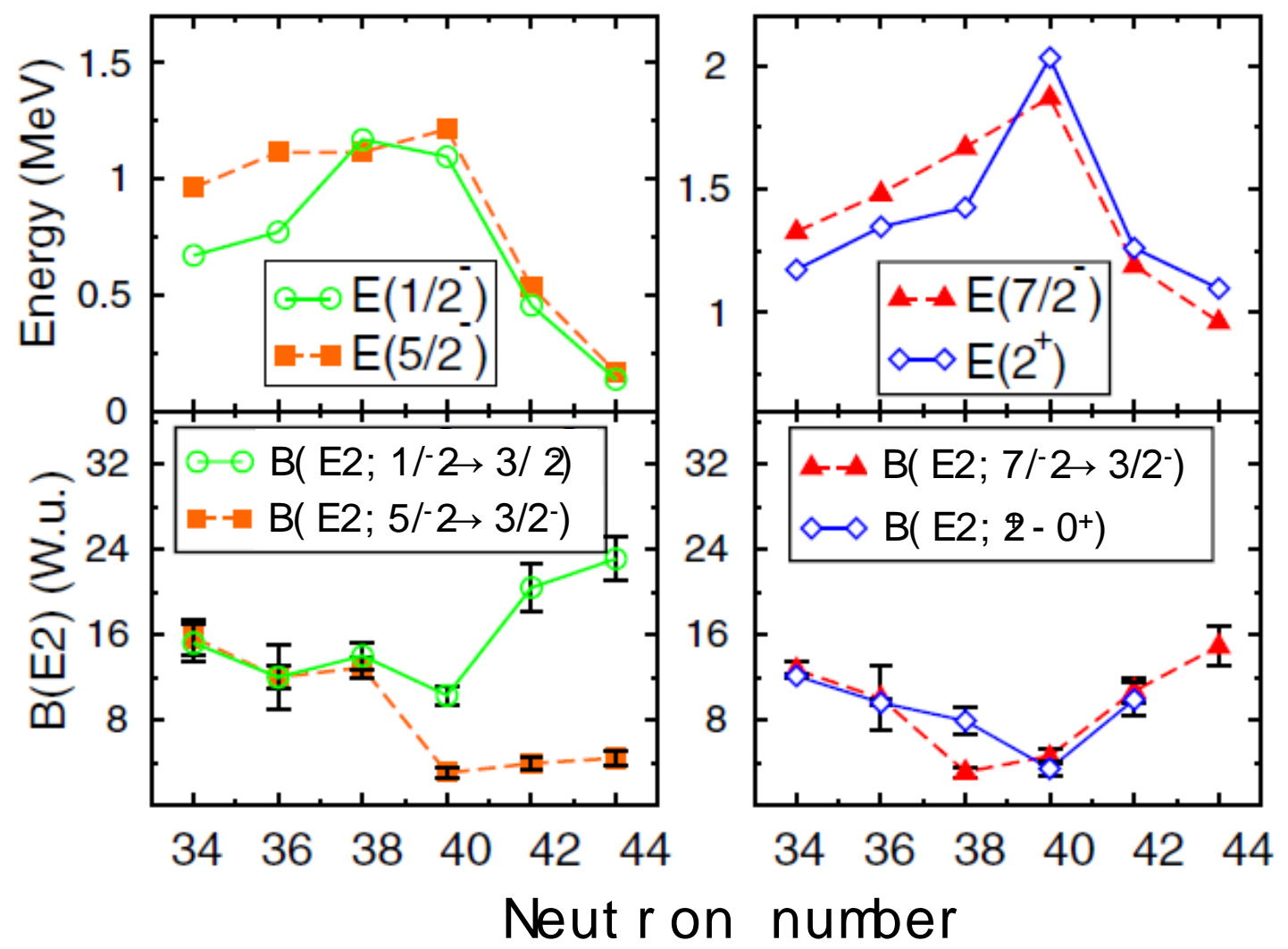

Figure 9. Top: The systematics of the energies of the $1 / 2^{-}, 5 / 2^{-}$, and core-coupled $7 / 2^{-}$states in odd mass ${ }^{63-73} \mathrm{Cu}$ along with the $2^{+}$levels in the corresponding eveneven ${ }^{62-72} N i$ are shown. Bottom: Experimental B(E2) values in ${ }^{63-73} \mathrm{Cu}$ and ${ }^{62-72} \mathrm{Ni}$. Note the similarity between the energies and B(E2)-values of the $7 / 2^{-}$and $2^{+}$states (right), the abrupt change in structure of the lowest lying $5 / 2^{-}$state and the increase in collectivity of the $1 / 2^{-}$state at $\mathrm{N}=40$ (adapted from [85]) (colour online).

reproduce the experimental $\mathrm{B}(\mathrm{E} 2)$-value recently obtained in ${ }^{80} \mathrm{Zn}$ (see further) obtains a better agreement indicating the importance of proton excitations across $Z=28$ to explain the collective structures observed in the neutron-rich copper isotopes [88]. Explaining the presence of spherical single-particle states, core-coupled states and collective states in the low-energy part of the isotopes at and around $\mathrm{Z}=28$ remains a challenge for theory and will have an impact on our understanding of the structure of the doubly magic ${ }^{78} \mathrm{Ni}$. For completeness, we note that very recently the ground state spin and parity of ${ }^{75} \mathrm{Cu}$ has been determined by collinear laser spectroscopy to be $5 / 2^{-}$confirming the anticipated crossing of the $5 / 2^{-}$and $3 / 2^{-}$states [89]. The latter measurements made it possible to propose a level scheme for ${ }^{75} \mathrm{Cu}$ [88].

The Coulomb experiments using the neutron-rich ${ }^{74,76,78,80} \mathrm{Zn}$ beams allowed to determine the first excited $2^{+}$state energy and the $B\left(E 2: 0^{+}-2^{+}\right)$in the semi magic ${ }^{80} \mathrm{Zn}(N=50)$ nucleus only two neutrons away from ${ }^{78} \mathrm{Ni}$. The $\gamma$-ray spectrum obtained after Coulomb excitation of a ${ }^{80} \mathrm{Zn}$ beam with an intensity of $\sim 3000$ particles per second accumulated for $\sim 100 \mathrm{~h}$ is shown in figure 10 . The spectrum shows what can be achieved 


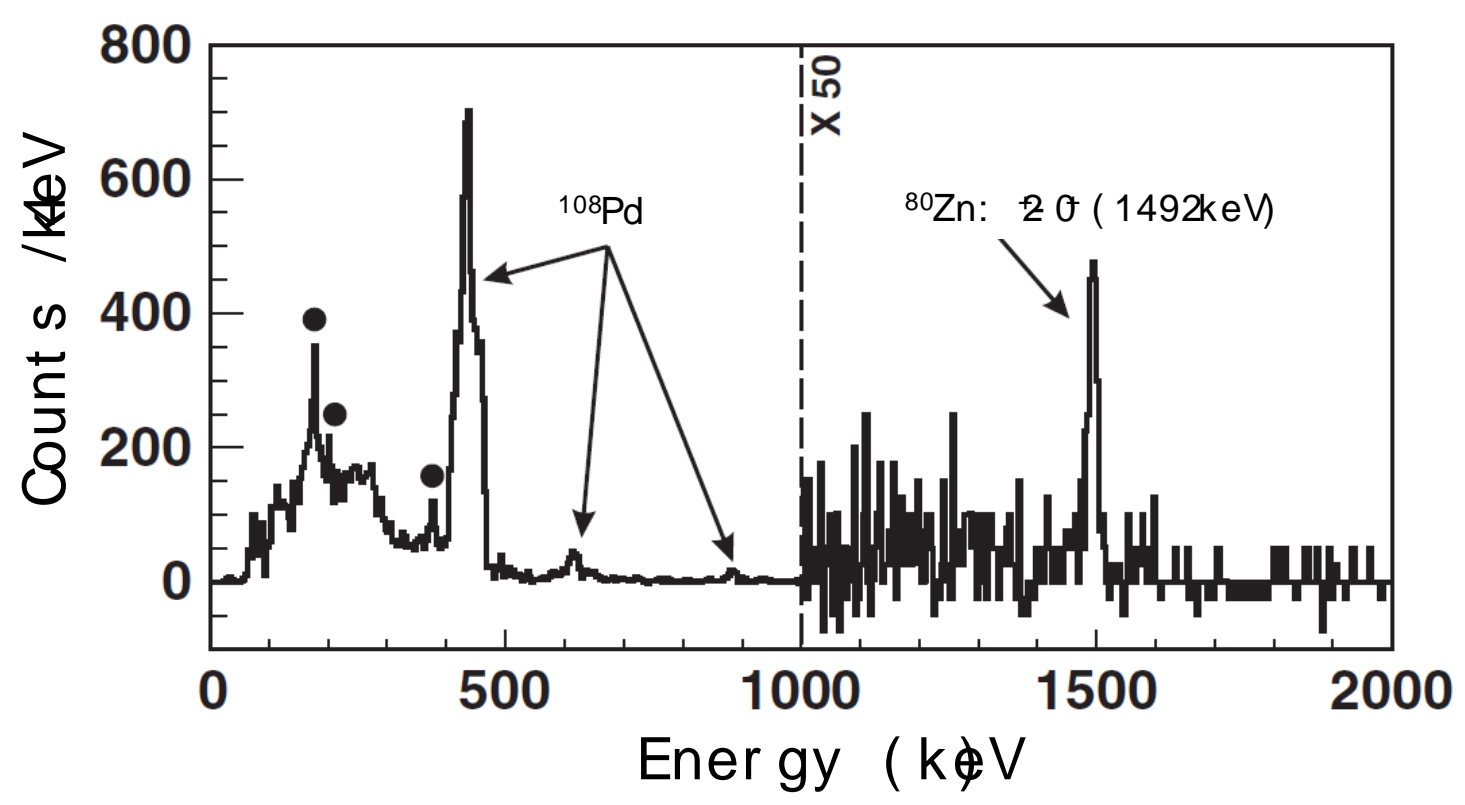

Figure 10. The Doppler corrected $\gamma$-ray spectrum for beam particle detection obtained after Coulomb excitation of a $2.79 \mathrm{MeV} / \mathrm{u}^{80} \mathrm{Zn}$ beam with an intensity of $\sim 3000$ particles per second on a $2 \mathrm{mg} / \mathrm{cm}^{2}$ thick ${ }^{108} P d$ target is shown. The $2^{+}-0^{+}$transition in ${ }^{80} \mathrm{Zn}$ and known transitions in ${ }^{108} \mathrm{Pd}$ are indicated together with transitions from beam contaminant ${ }^{80} R b$ (circles) (adapted from [26]).

with Coulomb excitation experiments using weak radioactive ion beams. Figure 14 of [27] shows the systematics of the $2^{+}$energy and the $B\left(E 2: 0^{+}-2^{+}\right)$value for the $\mathrm{N}=50$ isotones compared to predictions from shell-model calculations using ${ }^{56} \mathrm{Ni}$ as a core. The shell-model calculations assume only protons as active valence particles and a good agreement with the data is obtained provided large effective charges are used. This indicates a good $N=50$ shell closure and an important $Z=28$ core polarization around ${ }^{78} \mathrm{Ni}$. However, none of the present shell-model calculations using ${ }^{56} \mathrm{Ni}$ as a core reproduce the energy of the first excited $2^{+}$state in the neutron-rich zinc isotopes beyond $\mathrm{N}=40$ properly, while the $\mathrm{B}(\mathrm{E} 2)$-values are in reasonable agreement with experiment for the most exotic ones (see e.g. figure 8 from [62] and figure 13 from [27]). This puzzle remains to be solved.

Recently, one-neutron transfer reaction studies were also performed using a beam of ${ }^{66} \mathrm{Ni}$ at $3 \mathrm{MeV} / \mathrm{u}$. A multitude of states up to $6 \mathrm{MeV}$ excitation energy were populated in the $d\left({ }^{66} N i, p\right){ }^{67} N i$ reaction, some of then exhibiting a strong ground-state gamma transition. The results, currently under analysis, will reveal spins and parities, cross sections and decay patterns of the populated levels. The high intensity of the ${ }^{66} \mathrm{Ni}$ beam (a few times $10^{6}$ particles per second) and the high purity $(>95 \%$ ) will enable the study of the $t\left({ }^{66} N i, p\right){ }^{68} N i$ reactions, probing explicitly neutron pairing properties in ${ }^{68} \mathrm{Ni}$. 
4.2.3. The tin region: towards ${ }^{100} S n$ and around ${ }^{132} S n$ The tin nuclei with the closed $\mathrm{Z}=50$ proton shell, are notorious examples of nuclei that can be described using the general seniority scheme. For example the excitation energy of the first excited $2^{+}$is nearly constant throughout the whole isotopic chain from $\mathrm{N}=52$ to 80 . In this scheme the $B\left(E 2: 0^{+}-2^{+}\right)$value is expected to show a parabolic behavior with a maximum value mid-shell between $\mathrm{N}=50$ and 82 and zero in ${ }^{100} \mathrm{Sn}$ and ${ }^{132} \mathrm{Sn}$. The parabolic trend of the $B\left(E 2: 0^{+}-2^{+}\right)$is also predicted by large-scale shell-model calculations and was indeed nicely confirmed by an extended set of data on the neutron-rich tin isotopes [90]. Recent results from intermediate energy Coulomb excitation experiments, however, seem to deviate from this trend. In order to confirm and to extend these findings to nuclei closer to ${ }^{100} \mathrm{Sn}$ beams of ${ }^{106,108,110} \mathrm{Sn}$ were used to perform Coulomb excitation measurements at so-called 'safe energies' [91, 92]. The results clearly show a deviation from the parabolic trend of the $B\left(E 2: 0^{+}-2^{+}\right)$and an increase in transition strength when going to lighter nuclei approaching ${ }^{100} \mathrm{Sn}$. Using different nucleon-nucleon interactions in a large scale shell-model approach could not reproduce these findings. Along the same lines, experiments were performed using ${ }^{100,102,104} C d(\mathrm{Z}=48)$ [93]. These experimental findings clearly request further theoretical investigations of the structure around ${ }^{100} S n$.

Neutron-rich nuclei around ${ }^{132} S n$ have also been studied using Coulomb excitation in order to investigate the influence of the $\mathrm{N}=82$ and $\mathrm{Z}=50$ shell closures. Data were obtained with beams from even mass ${ }^{122-126} C d,{ }^{138-144} X e$ and ${ }^{140,142} B e$ isotopes [94]. These experiments were partially triggered by a surprising result obtained at the Oak-Ridge radioactive beam facility [95, 96]. Experiments using neutron-rich tin and tellurium beams in the vicinity of ${ }^{132} \mathrm{Sn}$ showed a deviation from the simple picture that the product of the energy of the first $2^{+}$state with $\mathrm{B}(\mathrm{E} 2)$-value can be described by a simple formula, the so-called Grodzins' formula [97]. The data are currently under analysis, but the new data combined with recent life-time measurements should resolve this issue.

\subsection{Shapes and shape coexistence}

Coulomb excitation is an ideal probe to investigate shapes and shape coexistence phenomena (see e.g. [98]). Beams from REX-ISOLDE have been used in different regions of the nuclear chart along $\mathrm{N}=20$ (see 4.2.1), along the $\mathrm{N}=\mathrm{Z}$ line, along the krypton and strontium isotopic chain where abrupt changes in the ground state deformation are observed and in the neutron-deficient region around $\mathrm{Z}=82$. These data can be used to test beyond mean-field calculations or symmetry-based models, and eventual deficiencies between data and calculation might highlight missing ingredients in the model (see e.g. [99, 100] and references therein). Here we limit ourselves to a short discussion on the ${ }^{70} \mathrm{Se}$ and the light mercury isotopes.

The Coulomb excitation cross section using a ${ }^{70} \mathrm{Se}$ beam on a ${ }^{104} \mathrm{Pd}$ was deduced and used to calculate the $B\left(E 2: 0^{+}-2_{1}^{+}\right)$. However, the final result on the latter depends 
on the diagonal matrix element $<2_{1}^{+}\|E 2\| 2_{1}^{+}>$. By including the life time of the $2_{1}^{+}$ state in the Coulomb excitation analysis, constraints on the sign and magnitude of the $<2_{1}^{+}\|E 2\| 2_{1}^{+}>$were put. Based on these findings, it was concluded that the ground state was, most likely, prolate [31]. However, a new measurement of the lifetime of the $2_{1}^{+}[101]$ was in strong disagreement with the literature value and the conclusion drawn by [31] had to be revised. The $2_{1}^{+}$of ${ }^{70} S e$, and most probably also its ground state, are of oblate nature in agreement with Hartree-Fock-Bogolyubov-based configuration-mixing calculations [101].

The neutron-deficient mercury isotopes and nuclei in the neighborhood of the $\mathrm{Z}=82$ closed proton shell with neutron number around $\mathrm{N}=104$, midshell between $\mathrm{N}=82$ and 126, are prototype examples of shape coexistence. One of the earliest experimental indications for shape coexistence came from the mean-square-charge-radii measurements using optical spectroscopy techniques, showing an strong shape staggering between the even-mass and odd-mass mercury isotopes with $A<186$ [102]. Meanwhile a wealth of data on mean-square-charge-radii in other isotopic chains, on coexisting rotational bands in several of these nuclei, on lifetimes of excited states, on fine-structure alphadecay data and on low-lying excited $0^{+}$states has been collected, with the discovery of triple shape-coexistence in the $\mathrm{N}=104$ nucleus ${ }^{186} \mathrm{~Pb}$ as probably the most intriguing aspect [103]. Little is known however on the matrix elements connecting the different states of the excited bands, nor on the sign of the deformation of the different bands. In order to shed light on these particular questions, Coulomb excitation experiments on the neutron-deficient mercury, lead, polonium and radon isotopes have recently been initiated. For these experiments mass $\sim 200$ beams were accelerated, a mass region that was far from being included in the initial REX-ISOLDE project. High quality data sets were obtained as can be seen in e.g. figure 11 that shows the particle gated gammaray spectrum obtained for ${ }^{182} \mathrm{Hg}$ [104]. The data are currently under analysis. The excitation of the $2_{1}^{+}, 2_{2}^{+}$and $4_{1}^{+}$is clearly observed. These first experiments clearly show the possibility to populate and study non-yrast states whose properties are very important to understand shape coexistence. It is interesting to note as well that a large amount of mercury X-rays were observed, indicating that the $0_{2}^{+}$state or that levels, decaying via strongly converted transitions, were populated. The latter is important as E0 transitions are considered to be fingerprints for shape coexistence. To pin down the final analysis and interpretation of these findings, the Coulomb excitation experiments are complemented with life-time and conversion-coefficient measurements [105].

\subsection{Other techniques}

In addition to the above mentioned program of Coulomb excitation and few-nucleon transfer reactions, other techniques have been and will be used at the REX-ISOLDE facility. The use of the transient field method in inverse kinematics to determine the sign and magnitude of the magnetic moment of the first excited $2^{+}$state in ${ }^{72,74} Z n$ is planned. These experiments can provide important insights into the wave function of these excited 


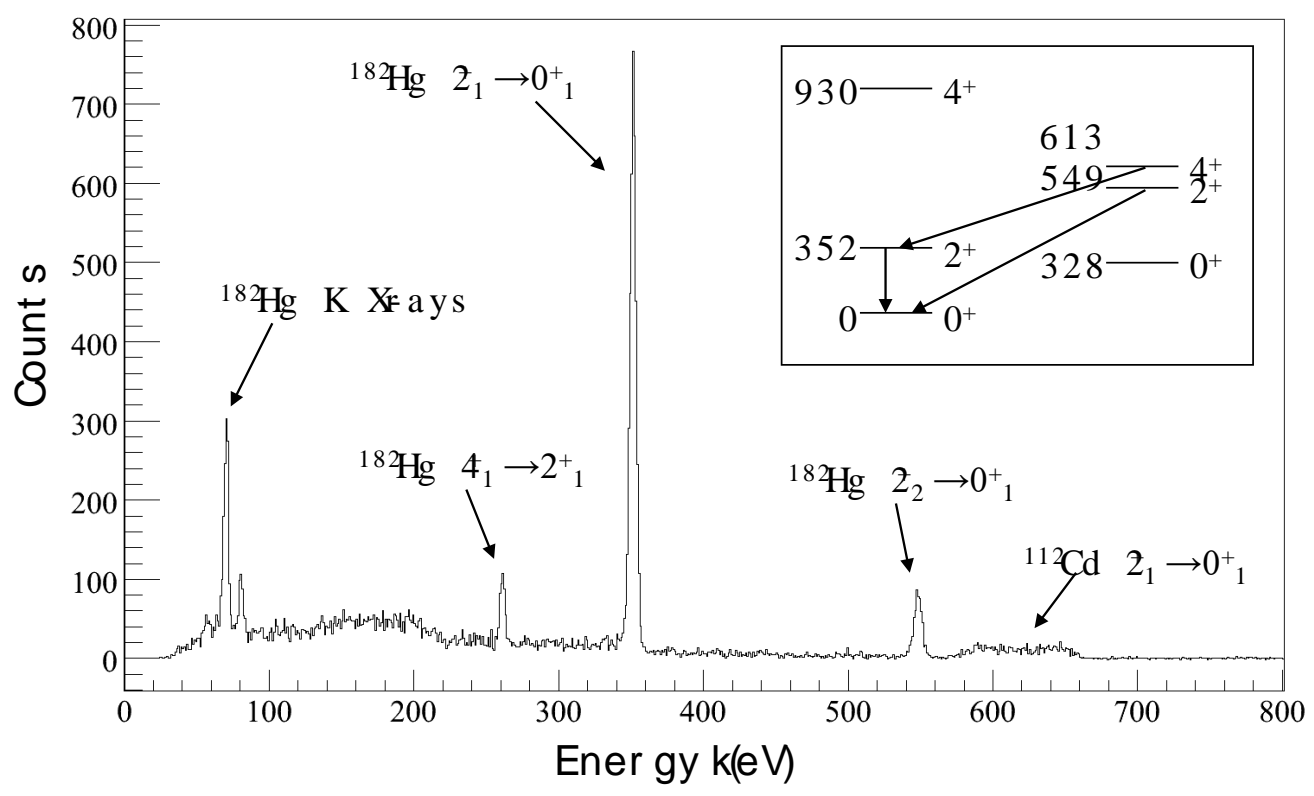

Figure 11. Part of the particle-gated gamma spectrum obtained after Coulomb excitation of ${ }^{182} \mathrm{Hg}$ on a ${ }^{112} \mathrm{Cd}$ target that was Doppler corrected for transitions in ${ }^{182} \mathrm{Hg}$. The insert shows the partial level scheme of ${ }^{182} \mathrm{Hg}$ where the observed transitions are indicated with an arrow. The broad bump around $620 \mathrm{keV}$ is the $2^{+}-0^{+}$transition in ${ }^{112} C d$ due to target excitation. The population of non-yrast states and an excess of $\mathrm{Hg} \mathrm{X}$-rays is observed. The former will be strongly enhanced with HIE-ISOLDE energies (see section 5).

states, for example regarding the importance of the neutron $g_{9 / 2}$ component in the zinc case [106]. Another technique that is currently under study is the so-called tilted foil technique to produce polarized beams [107]. The possible energy domains of the REX accelerator and its energy variability are ideally suited for this in-flight technique that, combined with a beta-NMR set-up, is especially interesting to study ground state magnetic or quadrupole moments of isotopes that are not readily amendable to laser techniques or have a very short half life. The optimum energy needed may vary from case to case, but varies from 0.1 up to about $1-2 \mathrm{MeV} / \mathrm{u}$, indeed an ideal window for the REX accelerator. The tilted-foil technique coupled to a beta-NMR set-up is also of interest for condensed matter research. The implantation of polarized radioactive probes in solid-state matrixes gives direct information on the electronic and lattice structure of the sample under study. In combination with the large variety of radioactive ion beams available, this potentially makes a very interesting research direction. Along the same lines, plans are under way to use the higher beam energy to obtain deeper implantation profiles to perform e.g. diffusion studies with conventional radioactive tracer techniques.

Finally, it is interesting to mention that modern Ge detector arrays are also reasonably efficient for neutron detection as demonstrated recently in a stable beam experiment [108]. The inelastic scattering of neutrons to the $0^{+}$excited state in ${ }^{72} \mathrm{Ge}$ 


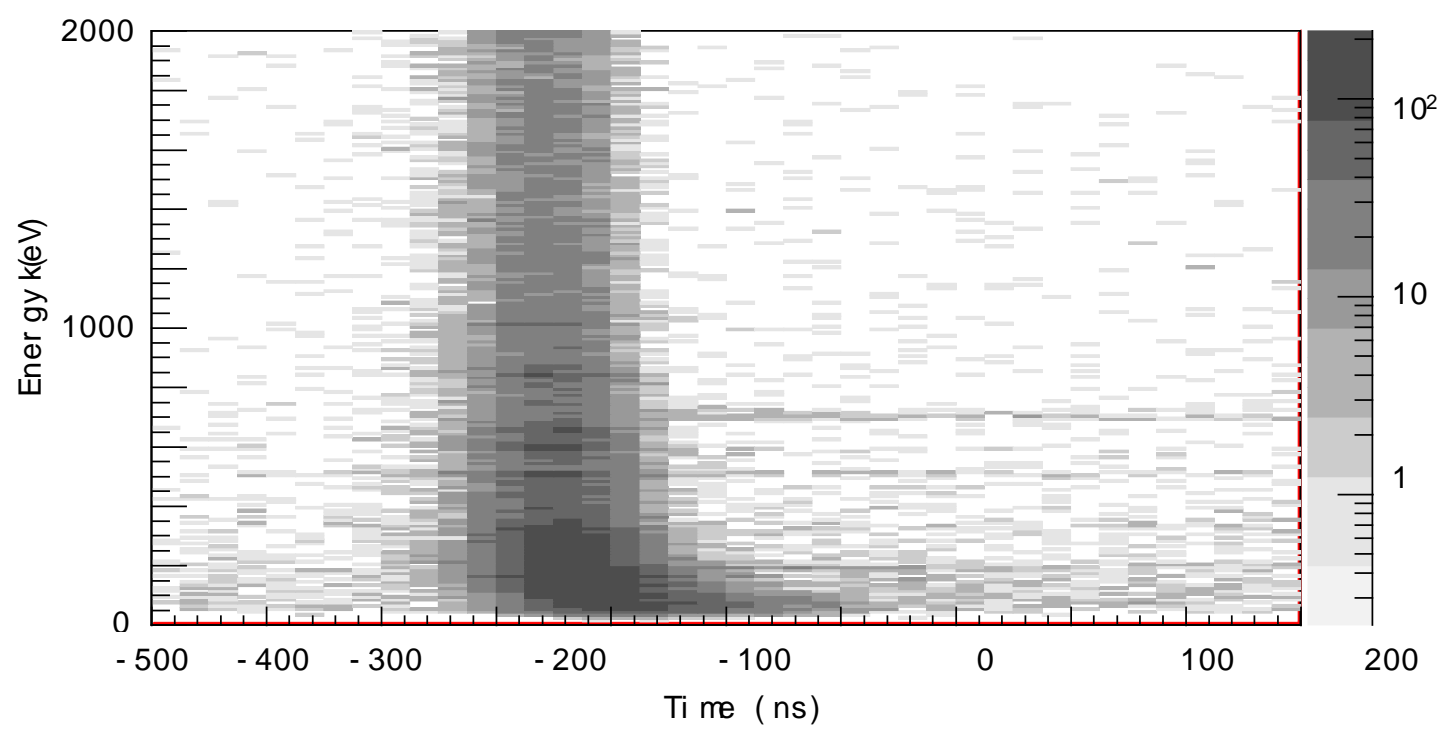

Figure 12. The energy recorded in Miniball for coincidences between a Miniball detector and a charged particle detector is shown versus the time difference between the two detectors. Prompt coincidences are situated around -150 ns. The line extending to the right at $690 \mathrm{keV}$ is due to neutrons from $\mathrm{d}\left({ }^{11} \mathrm{Be}, \mathrm{n}\right)$ events that are deteced in Miniball via ${ }^{72} \mathrm{Ge}\left(\mathrm{n}, \mathrm{n}^{\prime}\right)$ to the $444 \mathrm{~ns} 0^{+}$level. See the text.

that decays by emitting a $690 \mathrm{keV}$ conversion electron gives a particularly clear signal that will be recorded at the ${ }^{72} \mathrm{Ge}$ position in the crystal, i.e. the direction of the original neutron can be extracted (in contrast, its energy will not be recorded). It is remarkable that the detection conditions are sufficiently clean at REX-ISOLDE that this neutron signature can be identified even with a radioactive ${ }^{11}$ Be beam at $2.85 \mathrm{MeV} / \mathrm{u}$ of intensity about $10^{5}$ ions/s. As shown in figure 12 the neutron signature is visible already in the raw data set as slightly delayed coincidences between Miniball and the charged particle detector array.

\subsection{Astrophysics}

Experiments that are mainly motivated by astrophysics questions are a small component of the physics program at REX-ISOLDE compared to other facilities presented in this volume $[4,95,109]$. Some of the advancements of our knowledge on nuclear structure mentioned above will of course also improve the reliability of nuclear astrophysics calculations, see also [110]. Among the many facets of nuclear astrophysics [111], radioactive beams are mainly useful for explosive events [112] such as novae [113] or X-ray bursts.

The conditions for breakout of the hot CNO-cycle are still investigated for the latter two events, one of the key processes being the reaction ${ }^{14} \mathrm{O}(\alpha, \mathrm{p}){ }^{17} \mathrm{~F}$ that proceeds mainly through a resonance in ${ }^{18} \mathrm{Ne}$ at $6.15 \mathrm{MeV}$ excitation energy. A recent experiment at REX-ISOLDE [114] studied inelastic proton scattering of a post-accelerated ${ }^{17} \mathrm{~F}$ beam 
in order to determine more precisely the decay properties of the $6.15 \mathrm{MeV}$ state.

The inelastic proton branch, identified through coincidences between a proton group at $2.26 \mathrm{MeV}$ and $495 \mathrm{keV} \gamma$-rays detected in the Miniball array, was consistent with a strong inelastic proton branch approximately equal in intensity to the ground-state branch. Combining this result with other experiments [95] it appears that ${ }^{14} \mathrm{O}$ will be destroyed through the $(\alpha, \mathrm{p})$ reaction in X-ray bursts, but not in novae, and that the inelastic branch will indeed make a significant contribution.

A typical feature of this experiment is that information on the astrophysically interesting reaction is obtained indirectly since the direct reaction rates are small, although not as prohibitively small as for non-explosive burning. The limits on the available beam time at ISOLDE indicates that the main contribution of REX-ISOLDE in this field in the future will be for reactions with beams that are unique for ISOLDE or for reactions where superior selectivity is offered by the ISOLDE-REX-Miniball combination.

\section{Outlook and conclusion}

Although REX-ISOLDE soon can celebrate its 10th aniversary, we are really only at the beginning of the exploitation of the possibilities emerging by having post-accelerated beams at ISOLDE. By late 2009 REX-ISOLDE had accelerated 72 different radioactive isotopes (of 25 elements), which is no more than about $10 \%$ of the radioactive isotopes that ISOLDE can deliver.

There are several regions of the nuclear chart that are not yet explored like e.g. the rare earth region or the heavy mass region around and above ${ }^{208} \mathrm{~Pb}$. Among the subjects that new experiments currently are starting to look into are octupole collectivity with recent Coulomb experiments using beams as heavy as ${ }^{224} R a$, and mixed symmetry states, but many more remain.

An important lesson to remember for the future is that new techniques developed for measurements at low energy, typically those involving ion manipulation with traps, coolers and lasers but also other "tricks" that enable signal and background to be distinguished for weak beams, often can be applied directly at REX-ISOLDE or be adapted to the acceleration of radioactive beams. This cross fertilization between methods for production, acceleration and measurements of radioactive beams continues unabated and will be an important factor for the future competitiveness of the facility.

Another important factor for the future of REX-ISOLDE is of course upgrades of the machine. A general major upgrade of ISOLDE, the HIE-ISOLDE project [115, 116], has recently been approved at CERN. Improvements of the accelerated beams indeed constitute a major component of HIE-ISOLDE that also includes increases of the primary proton driver beam intensity and several improvements in the low-energy parts of ISOLDE, some of which will of course also lead to better accelerated beams. The plans are to replace the current linac with a superconducting linac that will be installed in stages (see figure 13). The aim of the first stage is to add cavities that will increase 


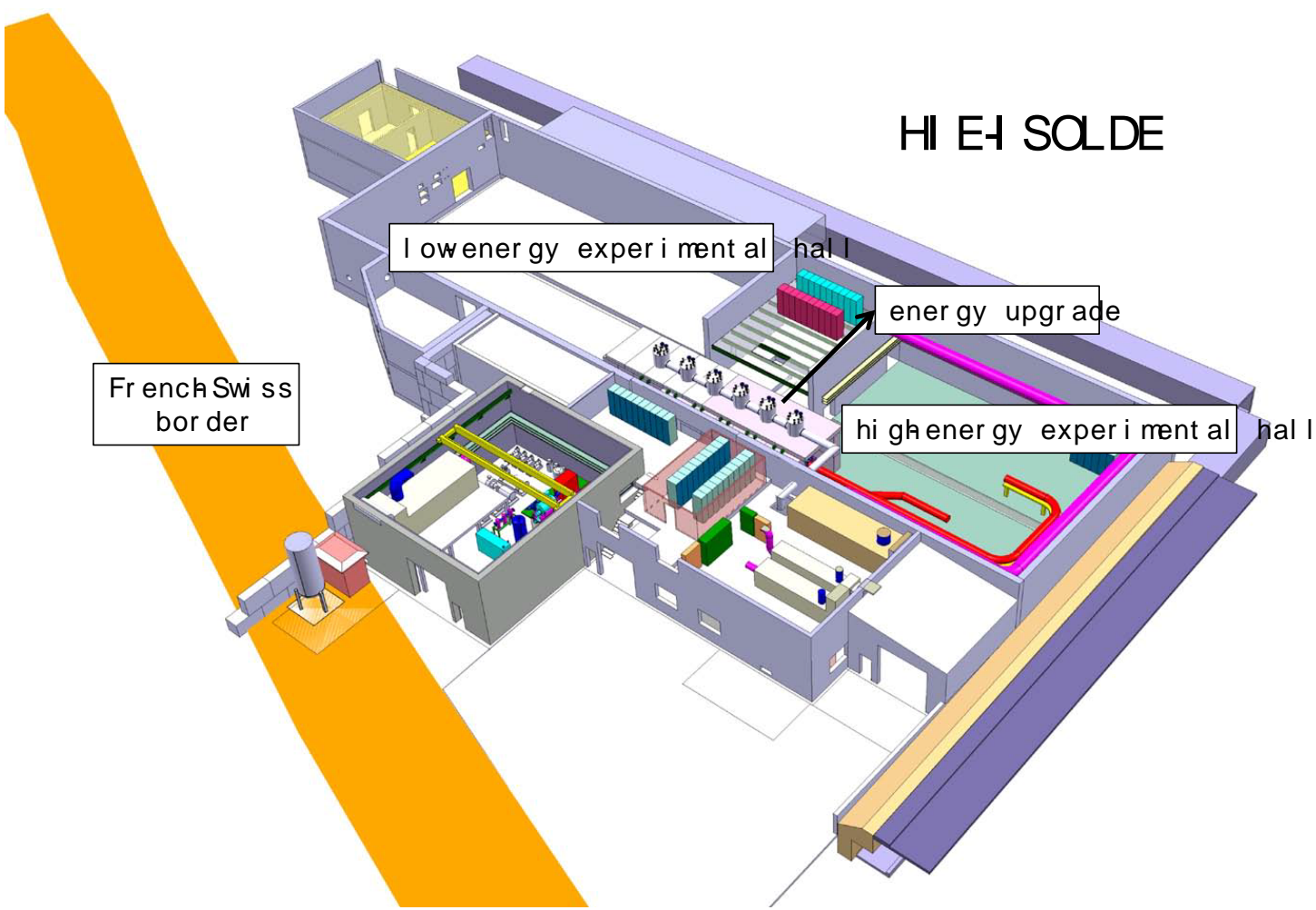

Figure 13. Lay-out of the HIE-ISOLDE experimental hall and service buildings. The addition of the super conducting cavities will bring the energy of ISOLDE's radioactive ion beams from the current $3 \mathrm{MeV} / \mathrm{u}$ up to $10 \mathrm{MeV} / \mathrm{u}$ (colour online).

the energy of the beams to $5.5 \mathrm{MeV} / \mathrm{u}$. This would allow multi-step Coulomb excitation to be performed efficiently for all radioactive beams that can be produced at ISOLDE; furthermore the conditions for transfer experiments would be improved. A second stage where the energy is raised to $10 \mathrm{MeV} / \mathrm{u}$ would enable more extensive studies involving transfer reactions also using heavy mass beams (A 200). Initial physics opportunities are described in [116] while recent updates are posted on the ISOLDE website [117]. The low energy stage of REX-ISOLDE is also planned to be upgraded so that charge breeding of the radioactive ions can take place faster thereby reducing the delay losses and increasing the efficiency in particular for heavy-mass accelerated radioactive beams.

\section{Acknowledgements}

We would like to acknowledge D. Voulot, F. Wenander, N. Bree, L. Acosta and J. Johansen for supplying us with unpublished data or allowing the use of unpublished figures. This work was supported by FWO-Vlaanderen (Belgium), GOA/2004/03 (BOFK.U.Leuven), the Interuniversity Attraction Poles Programme - Belgian Science Policy (BriX network P6/23).

\section{References}

[1] Blomqvist J 1967 Arkiv för fysik 36681

[2] Hansen PG 1996 History of CERN vol 3 ed J Krige (Amsterdam: North-Holland) p 327 
[3] Haas H, Ravn H, Schempp A, Allardyce BW, Jonson B and Rolfs C 1990 Proc. of the first Int. Conference on Radioactive Nuclear Beams (1989 Berkeley) ed WD Myers et al (Singapore: World Scientific) p 59

[4] Huyse $\mathrm{M}$ and Raabe $\mathrm{R}$ this volume (Louvain la Neuve contribution)

[5] Jonson B and Warner DD 1992 Int. workshop on the Physics and Techniques of Secondary Nuclear Beams (1992 Dourdan) ed JF Burandet et al (Gif-sur-Yvette: Editions Frontieres) p 355

[6] Forkel-Wirth D and Bollen G (eds) 2000 Hyperfine Interactions 1291

[7] Habs D et al 1994 Radioactive beam experiments at ISOLDE: Coulomb excitation and neutron transfer reactions of exotic nuclei Proposal to the ISOLDE committee CERN/ISC 94-25 ISC/P68

[8] Habs D et al 1998 Nucl. Instr. Methods B 139128

[9] Kester O et al 2000 Hyperfine Interactions 12943

[10] Kester O et al 2003 Nucl. Instr. Methods B 20420

[11] Cederkall J et al 2004 Nucl. Phys. A 74617

[12] Ames F, Cederkall J, Sieber T and Wenander F 2005 The REX-ISOLDE Facility: Design and Commissioning Report CERN report CERN-2005-009

[13] Voulot D, Wenander F, Piselli E, Scrivens R, Lindroos M, Jeppesen HB, Fraile LM, Sturm S, Delahaye P and the REX-ISOLDE collaboration 2008 Nucl. Instrum. Methods B 2664103

[14] Habs D et al 2000 Hyperfine Interactions 12943

[15] Mané E et al 2009 Eur. Phys. J. A42 503

[16] Kugler E 2000 Hyperfine Interactions 12923

[17] Mishin VI, Fedoseyev VN, Kluge HJ, Letokhov VS, Ravn HL, Scheerer F, Shirakabe Y, Sundell S, Tengblad O and the ISOLDE Collaboration 1993 Nucl. Instrum. Methods B 73550

[18] Köster U et al 2003 Nucl. Instrum. Methods B 204347

[19] Van Roosbroeck J et al 2004 Phys. Rev. Lett. 92112501

[20] Lettry J et al 1997 Nucl. Instrum. Methods B 126130

[21] Bree N et al 2008 Phys. Rev.C 78047301

[22] Herlert A et al 2006 International Symposium on Nuclear Astrophysics Nuclei in the Cosmos IX Proceedings of Science PoS(NIC-IX)051

[23] Ames F et al 2005 Nucl. Instrum. Methods A 53817

[24] Wolf B et al 2002 Rev. Scien. Instr. 73682

[25] Sieber T et al 2002 Nucl. Phys. A 701656

[26] Van de Walle E et al 2007 Phys. Rev. Lett. 99142501

[27] Van de Walle E et al 2009 Phys. Rev. C 79014309

[28] Catherall R et al 2003 Nucl. Instrum. Methods B 204235

[29] Köster U et al 2008 Nucl. Instrum. Methods B 2664229

[30] Van de Walle E et al 2009 Eur. Phys. J. A 42401

[31] Hurst AM et al 2007 Phys. Rev. Lett. 98072501

[32] Eberth J et al 2001 Progr. Part. Nucl. Phys. 46389

[33] Ostrowski A et al 2002 Nucl. Instrum. Methods A 480448

[34] Jeppesen HB et al 2005 Nucl. Phys. A 748374

[35] Tengborn E 2009 Transfer reactions in invers kinematics at REX-ISOLDE Ph.D. thesis (Chalmers University of Technology)

[36] Acosta L et al 2009 Eur. Phys. J. A42 461

[37] Tengblad O, Bergmann UC, Fraile LM, Fynbo HOU and Walsh S 2004 Nucl. Instrum. Methods A $\mathbf{5 2 5} 458$

[38] Bildstein V et al 2007 Progr. Part. Nucl. Phys. 59386

[39] Kröll Th et al 2009 Procedings of "Nuclear Structure and Dynamics '09 AIP Conf. Proceedings 1165363

[40] Jonson B 2004 Phys. Rep. 3891

[41] Okołowicz J, Płoszajczak and Rotter I 2003 Phys. Rep. 374271

[42] Jensen AS, Riisager K, Fedorov DV and Garrido E 2004 Rev. Mod. Phys. 76215 
[43] von Oertzen W, Freer M and Kanada-En'yo Y 2006 Phys. Rep. 43243

[44] Sorlin O and Porquet M-G 2008 Prog. Part. Nucl. Phys. 61602

[45] Jeppesen HB et al 2006 Phys. Lett. B 63517

[46] Jeppesen HB et al 2006 Phys. Lett. B 642449

[47] Audi G, Wapstra AH and Thibault C 2003 Nucl. Phys. A 729337

[48] Tilley DR, Kelley JH, Godwin JL, Millener DJ, Purcell JE, Sheu CG and Weller HR 2004 Nucl. Phys. A $\mathbf{7 4 5} 155$

[49] Kirsebom $\mathrm{O}$ et al 2010 in preparation

[50] Raabe R et al 2008 Phys. Rev. Lett. 101212501

[51] Kanungo R et al 2008 Phys. Lett. B 66026

[52] Guo B et al 2005 Nucl. Phys. A 761162

[53] Gade A et al 2008 Phys. Rev. C 77044306

[54] Lee J et al 2010 Phys. Rev. Lett. 104112701

[55] Lee J, Tostevin JA, Brown BA, Delaunay F, Lynch WG, Saelim MJ and Tsang MB 2006 Phys. Rev. C 73044608

[56] Timofeyuk NK 2009 Phys. Rev. Lett. 103242501

[57] Geithner W et al 1999 Phys. Rev. Lett 833792

[58] Fortier S et al 1999 Phys. Lett. B 46122

[59] Kanungo R et al 2010 Phys. Lett. B 682391

[60] Keeley N, Raabe R, Alamanos N and Sida JL 2007 Prog. Part. Nucl. Phys. 59579

[61] Di Pietro A et al 2010 Phys. Rev. Lett. 105022701

[62] Honma M et al 2009 Phys. Rev. C 80064323

[63] Sieja K et al 2009 Phys. Rev. C79 064310

[64] Otsuka T et al 2010 Phys. Rev. Lett. 104012501

[65] Smirnova NA et al 2010 Phys. Lett. B 686109

[66] Yordanov DT et al 2007 Phys. Rev. Lett. 99212501

[67] Gade A et al 2007 Phys. Rev. Lett. 99072502

[68] Motobayashi T et al 1995 Phys. Lett. B 3469

[69] Terry JR et al 2008 Phys. Rev. C 77014316

[70] Caurier E et al 1998 Phys. Rev.C 582033

[71] Otsuka T et al 2001 Phys. Rev. Lett. 87082502

[72] Niedermaier O et al 2005 Phys. Rev. Lett. 94172501

[73] Scheit $\mathrm{H}$ et al 2010 to be published

[74] Scheit H et al 2004 Nucl. Phys. A 746 96c

[75] Niedermaier $\mathrm{O}$ et al 2005 PhD Thesis University of Heidelberg (http://www.ub.uniheidelberg.de/archiv/5647/)

[76] Wimmer K et al 2010 Phys. Rev. Lett. submitted for publication

[77] Blank B 2009 The Euroschool Lectures on Physics with Exotic Beams, Vol. III, Lect. Notes Phys. (Springer, Berlin Heidelberg) $\mathbf{7 6 4} 153$

[78] Lisetskiy AF et al 2003 Phys. Rev.C 68034316

[79] Honma M et al 2004 Phys. Rev.C 69034335

[80] Hosmer PT et al 2005 Phys. Rev. Lett. 94112501

[81] Van Roosbroeck J et al 2004 Phys. Rev.C 92112501

[82] Pauwels D et al 2008 Phys. Rev.C 78 041307(R)

[83] Ferrer R et al 2010 Phys. Rev.C 81044318

[84] Stefanescu I et al 2007 Phys. Rev. Lett. 98122701

[85] Stefanescu I et al 2008 Phys. Rev. Lett. 100112502

[86] Sorlin O et al 2002 Phys. Rev. Lett. 88092501

[87] Franchoo S et al 1998 Phys. Rev. Lett. 813100

[88] Daugas JM et al 2010 Phys. Rev. C 81034304

[89] Flanagan KT et al 2009 Phys. Rev. Lett. 103142501 
[90] Banu A et al 2005 Phys. Rev.C 72 061305(R)

[91] Ekström A et al 2008 Phys. Rev. Lett. 101012502

[92] Cederkall J et al 2007 Phys. Rev. Lett. 98172501

[93] Ekström A et al 2009 Phys. Rev.C 80054302

[94] Kröll T et al 2008 International Conference on Frontiers in Nuclear Structure, Astrophysics and Reactions (FINUSTAR) CP1012 American Institute of Physics 84

[95] Beene, Gross, Nazarewicz, Stracener and Tatum this volume (HRIBF contribution)

[96] Radford D et al 2002 Phys. Rev. Lett. 88222501

[97] Grodzins L et al 1962 Phys. Lett.B 288

[98] Görgen A et al 2010 J. Phys. G: Nucl. Part. Phys. 37103101

[99] Sabbey B et al 2007 Phys. Rev.C 75044305

[100] Hellemans V et al 2005 Phys. Rev.C 71034308

[101] Ljungvall J et al 2008 Phys. Rev. Lett. 100102502

[102] Bonn J et al 1972 Phys. Lett. B 38308

[103] Andreyev AN et al 2000 Nature 405430

[104] Petts A et al 2009 Capture Gamma-Ray Spectroscopy and Related Topics CP1090 American Insitute of Physics 978414

[105] Grahn A et al 2009 Phys. Rev.C 80014324

[106] Jungclaus A et al 2008 Proposal to the ISOLDE committee CERN/INTC IS483

[107] Lindroos M et al 2000 Hyperfine Interactions 129109

[108] Jenkins DG, Glover R, Herzberg RD, Boston AJ, Gray-Jones C and Nordlund A 2009 Nucl. Instr. Methods A 602457

[109] Ball $\mathrm{G}$ this volume (ISAC contribution)

[110] Grawe H, Langanke K and Martínez-Pinedo 2007 Rep. Prog. Phys. 701525

[111] Langanke K, Thielemann F-K and Wiescher M (eds) 2006 Special issue on Nuclear Astrophysics Nucl. Phys. A 7771

[112] Woods PJ 2009 Nucl. Phys. A 827 582c

[113] José J and Hernanz M 200734 R431

[114] He JJ et al 2009 Phys. Rev. C 80042801

[115] Lindroos M and Nilsson T (eds) 2006 HIE-ISOLDE: the technical options CERN report CERN2006-013

[116] Riisager K, Butler P, Huyse M and Krücken R (eds) 2007 HIE-ISOLDE: the scientific opportunities CERN report CERN-2007-008

[117] ISOLDE website 2010 http://isolde.web.cern.ch/ISOLDE/ 